\title{
19. SEDIMENT CONSOLIDATION AND PERMEABILITY AT THE BARBADOS FOREARC
}

\author{
Elliott Taylor ${ }^{2,3}$ and John Leonard ${ }^{4}$
}

\begin{abstract}
Sediments cored from the Barbados Ridge accretionary complex at ODP Sites 671, 672, and 676 are progressively underconsolidated with depth. Very low measured intergranular permeabilities contribute substantially to the probable excess pore-water pressures that account for the underconsolidated behavior. The estimated pore pressures immediately overlying the incipient décollement at the reference site are nearly equal to the overburden stress. Data show that the pore fluids within the fine-grained sediments of the complex are absorbing the tectonic shock of underthrusting as they are accreted, effectively leading to "lubricated subduction" of the underthrust Oligocene and older sediments. Lateral compression and dewatering by fracture permeability is taking place in the accreted section despite apparent underconsolidation, resulting in limited pore-volume reduction and sediment strengthening from east to west on the ridge.
\end{abstract}

\section{INTRODUCTION}

An extensive program of physical properties measurements and geotechnical sampling of cores from the Barbados Ridge accretionary prism was carried out during Ocean Drilling Program Leg 110. The results from shore-based laboratory consolidation and intergranular permeability tests are reported in this paper and discussed along with pertinent data collected aboard the drillship.

Leg 110 was the second scientific drilling cruise to the Lesser Antilles forearc/Barbados submarine ridge area (Fig. 1). This location was drilled for the first time by the Glomar Challenger during Leg 78A of the Deep Sea Drilling Project. The results of this cruise suggested that high fluid pressures are found along the décollement zone separating the underthrusting Atlantic Ocean crust and the overriding Carribean Plate, but drilling failed to penetrate this major fault surface (Biju-Duval, Moore, et al., 1984). Results of previous DSDP active-margin cruises on the trenches rimming the Pacific have indicated that the physical properties of sediments at convergent margins are affected to different degrees by the tectonic stresses associated with plate subduction and sediment accretion (Shephard and Bryant, 1983; von Huene and Lee, 1983; Moore, 1986). Fluid flow, thermal conditions, and high pore-water pressures beneath the landward trench slope each appear to exert, in turn, a major control on accretionary wedge growth in these areas (Westbrook and Smith, 1983; Davis and von Huene, 1987). The Lesser Antilles convergent margin represents a type section for a wedge of accreted sediment located in a forearc region where the different mechanisms of offscraping at the prism toe, underplating at the base, thrust faulting, dewatering, and deformation inside the wedge can be observed within a zone of relatively slow $(1-2 \mathrm{~cm} / \mathrm{yr})$ convergence.

A total of nine sites have now been drilled along an east-west transect $23 \mathrm{~km}$ long, at about $15^{\circ} 30^{\prime} \mathrm{N}$, across the outer deformation front marking the eastern toe of the Lesser Antilles accretionary prism. DSDP Leg 78A cored Sites 541,542 , and 543, while ODP Leg 110 added six more sites, 671 through 676 (Mascle, Moore, et al., 1988). Each Leg included a reference site,

\footnotetext{
${ }^{1}$ Moore, J. C., Mascle, A., et al., 1990. Proc. ODP, Sci. Results, 110: College Station, TX (Ocean Drilling Program).

2 Ocean Drilling Program, Texas A\&M University, College Station, TX 77840.

3 Now at School of Oceanography, WB-10, University of Washington, Seattle, WA 98195 .

4 Dept. of Oceanography, Texas A\&M University, College Station, TX 77840.
}

Sites 543 and 672 , which were drilled 3.5 and $6 \mathrm{~km}$, respectively, east of the deformation front. The reference sites are located on the Atlantic plate where sediments are expected to be principally influenced by normal vertical compaction and undisturbed by subduction or accretion processes. At the other sites, sediments are progressively more deformed from east to west as they are scraped off in packets from the underriding Atlantic lithosphere to the overriding toe of the accretionary prism.

Sediment physical properties were measured in the shipboard laboratory, and 20 whole-round samples from cores at four sites $(671,672,673$, and 676$)$, were taken for this shore-based investigation. All whole-round samples are of very good to excellent quality and showed almost no visible signs of drilling disruption or other disturbance. The geotechnical analysis of each site is discussed in sequence, moving east to west from the reference hole to the prism toe and up onto the sediment wedge, as tectonic deformation in the complex becomes progressively more intense.

\section{METHODS}

Extensive physical properties measurements were routinely performed aboard ship on all Leg 110 cores according to the general procedures outlined by Boyce (1976). The parameters measured are index properties (wet and dry bulk density, water content, porosity), vane shear strength, compressional wave velocity, thermal conductivity, and formation factor. Shore-based laboratory analyses consisted of a total of 30 vertical and horizontal consolidation and permeability tests on all whole-round core samples, using seven back-pressured Anteus consolidometers, a new GDS computerized continuous gradient machine, a modified Geotest consolidometer (Silva et al., 1983), and two Soil Test standard deadload oedometers. The Anteus and Soil Test devices were fitted for falling head permeability measurements. Nearly all tests were backpressured as recommended by Lowe et al. (1964) to insure complete saturation of the sediment. All testing techniques were standard soil mechanics procedures, as described in Lambe (1951), Head (1982), and Bowles (1978). With the exception of five consolidation tests run on the continuousgradient GDS machine, tests on samples as deep as 213 meters below sea floor (mbsf) were step-loaded in multiples of two, starting at $3 \mathrm{kPa}$ up to a maximum load of $3200 \mathrm{kPa}$. Two very deep samples from $454 \mathrm{mbsf}$ were loaded to $8000 \mathrm{kPa}$ on the Soil Test consolidometers. Falling-head permeability measurements, herein described in terms of hydraulic conductivity or coefficient of permeability (k), were obtained at least $24 \mathrm{hr}$ after the application of each new load on the Anteus devices, yielding a distribution of permeability values at incremental void ratios for most of the samples. Values of permeability computed from the rate of consolidation response to loading (Lambe and Whitman, 1969) were also obtained, though these were typically one order of magnitude lower than the direct measurements. Some constant head permeability tests were carried out at low hydraulic gradients as described by Silva et al., 


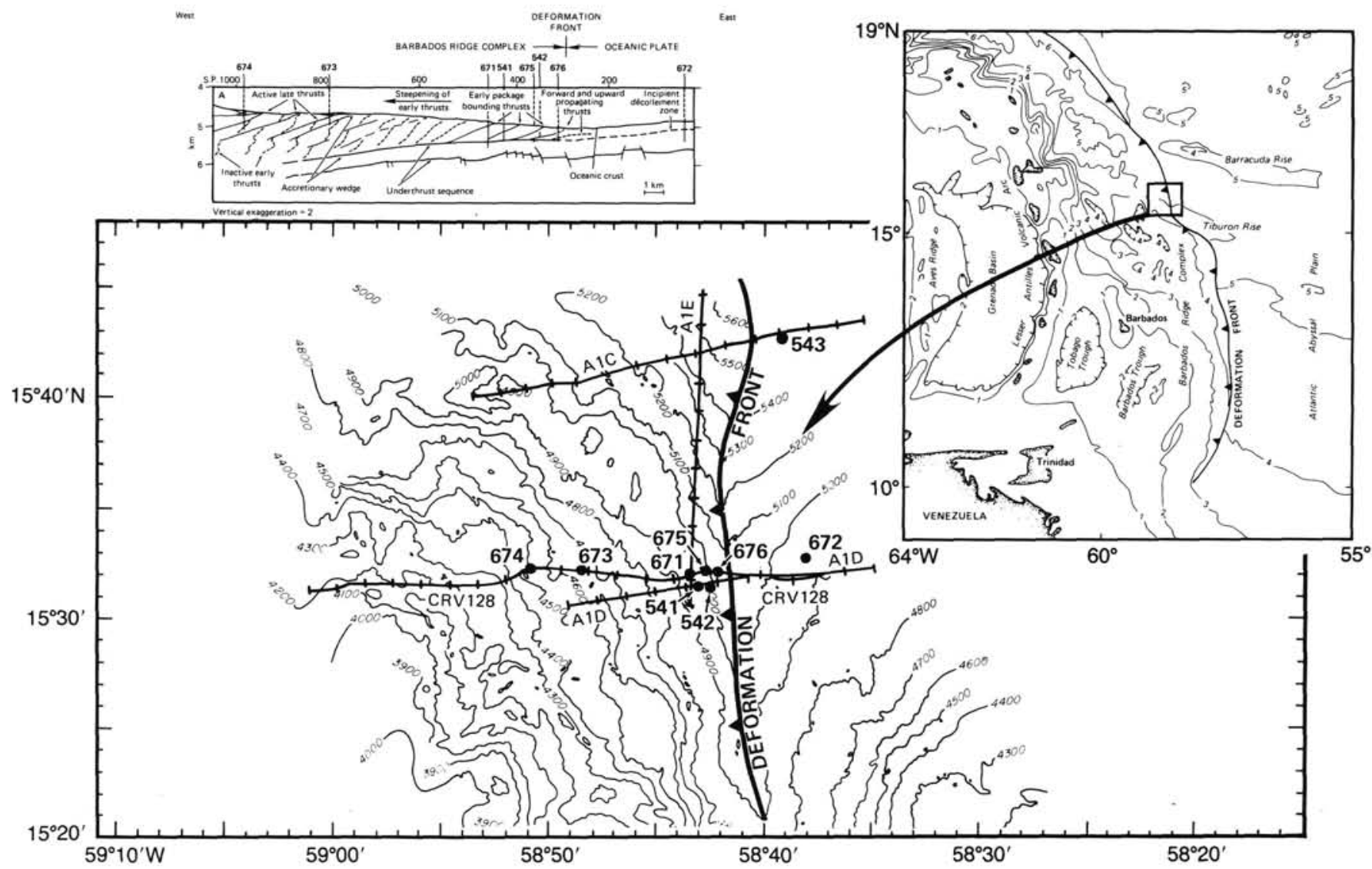

Figure 1. Location of ODP Leg 110 and DSDP Leg 78A drill sites across the Barbados forearc. Bathymetric contours are in 1000-m intervals and cross-section is line CRV 128 (from Mascle, Moore, et al., 1988).

1981. Differences to the Silva et al., procedures were: (1) the samples were tested under triaxial stress conditions, and (2) precise flow pumps were used to apply hydraulic gradients. Values of $\mathrm{k}$ are reported in $\mathrm{cm} / \mathrm{s}$, where 1 millidarcy is equivalent to $10^{-6} \mathrm{~cm} / \mathrm{s}$.

The state of consolidation of a sediment sample is obtained from the plotted curves of void ratio vs. effective overburden stress $\left(e-\log P_{o}^{\prime}\right) . P_{o}^{\prime}$ the present in-situ effective overburden, is computed by integrating the bulk densities from the seafloor (zero) to the sample depth $(z)$ and subtracting the hydrostatic head within that same interval. Casagrande's technique (1936) was used to determine the maximum preconsolidation stress $\left(P_{c_{\text {max }}}^{\prime}\right)$ and the minimum preconsolidation stress $\left(P_{c_{\min }}^{\prime}\right)$ was determined following Bryant, et al. (1986a). The preconsolidation stress is normally interpreted to be the maximum effective overburden load the sediment had experienced prior to laboratory loads. Sediments are termed normally consolidated if the present effective overburden is the greatest stress ever imposed, and $P_{o}^{\prime}$ equals $P_{c}^{\prime}$. If the sediment behaves as though it had come to equilibrium under a greater overburden stress at some time in the past, $P_{c}^{\prime}$ is greater than the current $P_{o}^{\prime}$ and the sample is termed overconsolidated. If $P_{c}^{\prime}$ is less than $P_{o}^{\prime}$, however, the sediment is termed underconsolidated, a condition usually attributed to excess porewater pressure supporting a portion of the sediment load at depth. The ratio of $P_{c}^{\prime}$ to $P_{o}^{\prime}$, the overconsolidation ratio (OCR), is generally used to describe the relative state of consolidation. Because this ratio is quite sensitive at low values of $P_{o}^{\prime}$, and is less indicative of relative underconsolidation than overconsolidation, the difference between $P_{c}^{\prime}$ and $P_{o}^{\prime}$, OCD, was used herein.

Although removing samples from in-situ stress conditions undoubtedly results in disturbance, oedometer tests can often provide an indication of the amount of apparent disturbance in a sample (Lambe and Whitman, 1969). A sharp reload to virgin curve transition of $e$-log $P_{o}^{\prime}$ curves is generally indicative of minimum disturbance. A rounded aspect of the reload curve would imply more disturbance. The samples tested appear to have undergone only slight disturbance. The horizontally tested samples were probably more disturbed during the laboratory sub-sampling than those tested vertically. All sub-samples were selected based on whole-core $\mathrm{x}$-radiographs to minimize known cases of sample disturbance. Some apparent disturbance in these samples may also be attributed to natural remolding, considering the tectonic environment from which they were extracted. The oedometer tests do provide, however, a good description of sediment void ratio-permeability and stressstrain relationships. The latter are expressed in terms of compression in$\operatorname{dex}, C_{c}$, where

$$
C_{c}=\frac{e}{\log P^{\prime}} .
$$

Other shore-based physical properties measurements performed on the whole-round samples include water content, grain-specific gravity, vane shear strength, Atterberg limits, grain-size analysis, and calcium carbonate content (dry wt. \%). The volumetric density determinations were made using air-helium gas comparison pycnometers. Initial void ratios were obtained from gravimetric/volumetric analyses that may differ slightly from apparent initial void ratios in $e$ - $\log P_{o}^{\prime}$ curves due to the lower accuracy of obtaining these values from oedometers. Calcium carbonate values were obtained using an optical coulometer. Vane shear measurements were made using a motorized vane at a shear rate of $60^{\circ}$ (1.05 radians) per min. Grain-size analyses were performed using the pipette method for silts and clays (Folk, 1974).

\section{RESULTS}

\section{Site 672}

Hole $672 \mathrm{~A}$, the single reference hole drilled on Leg 110 , is located $6 \mathrm{~km}$ east of the Barbados Ridge deformation front, or toe of the accretionary prism complex, in an area where the sediment cover on the Atlantic Plate is $800 \mathrm{~m}$ thick. Sediments at this site show no evidence in seismic records of lateral tectonic 
deformation from the subduction process, in stark comparison to those sediments observed in the accretionary complex to the west. Hole $672 \mathrm{~A}$ penetrated $500 \mathrm{~m}$ of Pleistocene to Eocene hemipelagic calcareous mud and claystones containing numerous ash layers (Mascle, Moore et al., 1988). Eight consolidation tests were performed on six whole-round core samples of light brownish and olive-gray calcareous mud and marl. Consolidation test results (Table 1 and Fig. 2) show that the near-surface, lower Pleistocene sample from 7.7 mbsf is overconsolidated; but in contrast the samples taken from depths of 55 to $213 \mathrm{mbsf}$ show progressively greater states of underconsolidation. The difference between $P_{c}^{\prime}$ and $P_{o}^{\prime}$, overconsolidation difference (OCD), decreases markedly from the overconsolidated condition of about +160 near the mudline to a very underconsolidated state of less than -900 in the lower Miocene-upper Oligocene sediments around $200 \mathrm{mbsf}$, the zone identified as the incipient décollement (Fig. 3). This zone is correlated with, and interpreted to be propagating east from, the deformation front.

Permeabilities interpolated at equivalent $P_{c}^{\prime}$ vertical loads and corrected for fluid viscosity at estimated in-situ temperatures are assumed to best approximate in-situ, intrinsic permeability $\left(k_{c}\right)$. Sediment permeabilities at Site 672 range from approximately $10^{-6}$ to $10^{-8} \mathrm{~cm} / \mathrm{s}$ (Fig. 3). Measured permeabilities decreased with increasing depth and with clay content. An unusually high $k_{c}$ was obtained for Sample 110-672A-2H-3 (145-150 $\mathrm{cm})$ at $7.7 \mathrm{mbsf}$ in the horizontal direction. A sand content over $20 \%$, the highest of any of the whole-rounds analyzed in this study, accounts for the higher permeability.

With only one exception, physical properties at this site show consistent downhole trends of decreasing porosity and water content and increasing density (Fig. 3). Grain-size analyses show that sand content is nearly zero below 90 mbsf. A silty clay texture predominates below $55 \mathrm{mbsf}$, with only one brief departure from an overall trend of increasing clay content. Sand intervals are again reached in the Oligocene-Eocene section below 300 mbsf. Atterberg limits show a pattern of increasing plasticity with depth. Almost all the Leg 110 samples tested classify as $\mathrm{CH}$ soils-inorganic clays of medium to high plasticity (Fig. 4) following the United Soils Classification System (Lambe and Whitman, 1969). Liquid limit $\left(w_{L}\right)$ and plastic index $\left(I_{p}\right)$ values at Site 672 increase with depth, and natural water contents are consistently between the liquid and plastic limits. X-ray diffraction analyses of sediment in this area have shown that the clay mineral content is dominated by smectite (montmorillonite), with lesser amounts of illite, kaolinite, and chlorite (Mascle, Moore, et al., 1988; Schoonmaker-Tribble, this volume). Quartz and feldspar are always present, but in minor quantities. Calcium carbonate is a principal sediment component from 0 to $124 \mathrm{mbsf}$, forming marl and calcareous mud. Clayey and siliceous mudstone intervals form lithologic Unit II to $227 \mathrm{mbsf}$ and are underlain by claystones, mudstones, and marlstones of variable carbonate content.

The most notable departure from the fairly uniform gradient of physical properties of Hole 672A occurs at $123 \mathrm{mbsf}$. For instance, Sample 110-672A-19X-3 (140-150 cm) (165.8 mbsf), of middle Miocene age, was extremely soft, easy to remold during Atterberg testing, and had an anomalously high water content of $120 \%$, which is closer to the liquid limit than other Site 672 samples. This sample is from the interval at Site 672 with the highest porosity measured; it was also the most underconsolidated (with an OCD of -916). These characteristics correlate well with the shipboard-defined index properties Zone 2 (lithologic Unit II equivalent) composed of smectitic and radiolarianrich mudstones.

Index properties return to a normal compaction trend below 207 mbsf, the start of index Zone 3 (lithologic Unit III equivalent), just above the Miocene/Oligocene boundary. Shore-based index properties measured on Sample 110-672A-24X-3 (140-150 $\mathrm{cm}$ ), from $213.3 \mathrm{mbsf}$, are consistent with the shipboard values for Zone 3. The shipboard data below this point then continue to vary regularly with depth. However, the consolidation test on Sample 110-672A-24X-3 (140-150 cm), shows that it is quite underconsolidated, although to a lesser degree than the lower half of index Zone 2, which marks the top of the incipient décollement. Interestingly, this deep sample also exhibits higher in-situ permeability, despite lower sediment porosity and a grain-size composition of no sand and $87 \%$ clay. This higher permeability may reflect fracture permeability related to localized scaly fabric. The higher measured permeability correlates with a chloride minimum and a methane peak that was observed by shipboard geochemists at $216 \mathrm{mbsf}$, suggesting horizontal advection of low-chloride waters at the base of the future décollement (Mascle, Moore, et al., 1988).

\section{Site 676}

Hole 676A was drilled $250 \mathrm{~m}$ arcward of the deformation front to explore the incipient stages of accretion. This single hole penetrated $310 \mathrm{~m}$ of hemipelagic lower Pleistocene-lower Miocene olive brown-gray calcareous muds, clays, marlstones, and volcanic ash layers. Eight consolidation tests were performed on four samples with each sample being tested in both the vertical and horizontal directions (Table 1 and Fig. 5). A comparison of $P_{o}^{\prime}$ and $P_{c}^{\prime}$ shows that the sediments are highly underconsolidated (Fig. 6). In contrast to equivalent lower Pleistocene surface sediments at Site 672 , there is no apparent overconsolidation near the surface. Permeabilities range from $10^{-7}$ to $10^{-8}$ $\mathrm{cm} / \mathrm{s}$, values slightly less than those at Site 672 (Fig. 6).

Hole 676A index properties are similar to those at Site 672, although the overall porosity, water content, and void ratio values are smaller at Site $\mathbf{6 7 6}$ and vane shear strengths are greater. Atterberg limits for Site 676 samples show a relatively high plastic index $\left(l_{p}\right)$ and a natural water content that approaches the plastic limit $\left(w_{p}\right)$ (Fig 6). The index properties reflect a structural repetition of units at $35 \mathrm{mbsf}$, the depth of the first imbricate thrust fault at the deformation front, but otherwise exhibit a systematic downhole trend. Permeabilities are nearly constant with increasing depth and clay content despite the larger sand fraction and calcium carbonate content. With the exception of the slight change in index properties just mentioned, there is no evidence in these data of the folding and steep bedding dips associated with the frontal thrust.

\section{Site 671}

Site 671 is significant in that it marks the first time the major fault surface (décollement) separating two plates in a subduction zone had been drilled. The site is located approximately $5 \mathrm{~km}$ behind the deformation front, and $11 \mathrm{~km}$ west of Site 672 . Cores recovered showed that the section there consists of about $500 \mathrm{~m}$ of an offscraped sequence of lower Pleistocene-lower Miocene, steeply dipping imbricate thrust packets of locally ashy, hemipelagic, olive-gray to yellowish calcareous mud(stone) to marl(stone) overlying siliceous to smectitic mud(stone). Below this lies a 40-m-thick décollement zone, characterized by shallow dip beds of scaly fabric. The lower $150 \mathrm{~m}$ cored is relatively undeformed, underthrust sediment of Oligocene age, composed of dark gray-green clay and mudstone with a cyclically variable carbonate content and a coarser-grained quartzose terrigenous component. Ten consolidation tests were performed on samples from depths ranging between 4.4 and 496 mbsf. All the samples tested were from the offscraped sequence, and results are given in Table 1 and Fig. 7.

A comparison of $P_{c}^{\prime}$ and $P_{o}^{\prime}$ shows that Sample 110-671A-1H-3 $(140-150 \mathrm{~cm})(4.4 \mathrm{mbsf})$, is overconsolidated $(\mathrm{OCD}=+15$ to +25 ) (Fig. 8). Sample 110-671B-3H-3 (140-150 cm) (21.3 mbsf), 
Table 1. Summary of ODP Leg 110 geotechnical and physical properties data from whole-round consolidation samples.

\begin{tabular}{|c|c|c|c|c|c|c|c|c|c|c|c|c|c|c|c|c|c|c|c|c|c|c|c|c|}
\hline Site-Hole & Core-Sect & MBSF & Lithology & 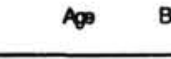 & $\begin{array}{l}\text { Bulk Dens } \\
\text { g/cc }\end{array}$ & $\begin{array}{c}\text { Water } \\
\%\end{array}$ & $\begin{array}{c}\text { Gin Dens } \\
\text { g/cc }\end{array}$ & $\begin{array}{l}\text { orosity } 1 \\
\%\end{array}$ & $\begin{array}{c}\text { Init Void } \\
\text { Ratio } \\
\end{array}$ & $\begin{array}{l}\mathrm{P} 0 \\
\mathrm{kPa}\end{array}$ & $\begin{array}{c}\mathrm{Pc}_{\max } \\
\mathrm{KPa}\end{array}$ & $\begin{array}{l}\mathrm{P} c \mathrm{~cm} \text { in } \\
\mathrm{kPa}\end{array}$ & $\begin{array}{l}\text { OverConso } \\
\text { Ditt max }\end{array}$ & $\begin{array}{r}10 C D \\
\times \quad \min \\
\end{array}$ & $\begin{array}{l}\mathrm{K} \text { at } \mathrm{Pc} \\
\mathrm{cm} / \mathrm{s}\end{array}$ & $\begin{array}{c}\text { Cc } \\
\text { index }\end{array}$ & $\begin{array}{c}\text { Vene Shear } \\
\mathrm{kPa}\end{array}$ & \multicolumn{3}{|c|}{ Grain Size \% } & $\begin{array}{l}\mathrm{CacOs} \\
\% \text { (ship) }\end{array}$ & $\begin{array}{l}\text { Attert } \\
\text { ) WL. }\end{array}$ & $\begin{array}{l}\text { rberg L } \\
. \text { Wp }\end{array}$ & Limits. \\
\hline & & & & & & & & & ÉCOL & EMEI & ENT SITE & & & & & & & & & & & & & \\
\hline $671 \mathrm{~A}$ & $1 \mathrm{H}-3$ & 4.41 & Marl/chalk/Ls & e. Pleistocene & 1.5 & 111 & 2.75 & 74 & 2.85 & 20 & 45 & 35 & 25 & 15 & $1.06 \mathrm{E}-06$ & 0.75 & 21.5 & 6.6 & 32.2 & 61.2 & & & & \\
\hline B & $3 \mathrm{H}-3 . \mathrm{T}$ & 20.95 & Cak mud & Pleis & 1.52 & 83 & 8 & 66.8 & & 105 & 140 & & 35 & & 8. & & 21.0 & 0.65 & 32.2 & 61.2 & 38.7 & $\begin{array}{l}128 \\
155\end{array}$ & $\begin{array}{r}42.1 \\
74\end{array}$ & $\begin{array}{r}86 \\
81\end{array}$ \\
\hline 6718 & $3 \mathrm{H}-3$ & 21.3 & Calc. $n$ & Pleistocene & 1.66 & 66.9 & 2.66 & 62 & 1.66 & 107 & 160 & 125 & 53 & 18 & $3.36 \mathrm{E}-07$ & 0.62 & 34 & 5.7 & 47.3 & 47.0 & 38.7 & 84 & 30.1 & 54 \\
\hline $671 B$ & $3 \mathrm{H}-4$ & 22.9 & Calc. mud & e. Pleistocene & 1.59 & 81.8 & 2.91 & 70. & 2.32 & 115 & 50 & 40 & .65 & .75 & & & 28.82 & & & & 38.7 & & & \\
\hline $671 B$ & $7 \mathrm{H}-3 . \mathrm{T}$ & 59.36 & Marl & late Pliocene & 1.73 & 48 & 2.94 & 58. & 1.42 & 346 & 166 & & .180 & & & & 18 & & & & 43.8 & 78 & 37 & 41 \\
\hline B & 77 & 60 & $\mathrm{Ma}$ & late PIK & 1.7 & 53 & 3 & 61 & 1.55 & 357 & 166 & & -191 & & & & & & & & 30 & 88 & 39 & 49 \\
\hline 6718 & $9 \mathrm{H}-2-\mathrm{V}$ & 76.8 & Marl & e. Pllocene & 1.77 & 52.9 & 2.81 & 58 & 1.41 & 468 & $400 \mathrm{~V}$ & $350 \mathrm{~V}$ & $-68 V$ & -118 & 8.57 & $0.5 \mathrm{~V}$ & 65 & 6.6 & 17.6 & 75.5 & 29.7 & 86.8 & 31.2 & 52 \\
\hline 6718 & 9 & & Mar & o. $P$ V & 1.77 & 52.9 & 29 & 58. & 1.41 & 469 & $750 \mathrm{H}$ & $720 \mathrm{H}$ & $281 \mathrm{H}$ & 251 & & $0.54 \mathrm{H}$ & 65 & 6.6 & 17.6 & 75.5 & 29.7 & 86.8 & 31.2 & 52 \\
\hline 6718 & $10 \mathrm{H}-3-\mathrm{T}$ & 87.79 & $\mathrm{Ma}$ & o. Pllox & 1.72 & 49 & .86 & B1. & 1.4 & 547 & 240 & & .307 & & & & 290 & & & & 41.3 & 101 & 49 & 52 \\
\hline $671 B$ & - $T$ & 89.33 & Marl & e. $\mathrm{Pl}$ & 1.71 & 54 & 85 & 58 & 1.26 & 558 & 240 & & .318 & & & & 195 & & & & 40.8 & 85 & 35 & 50 \\
\hline $671 B$ & $1:$ & 135.5 & Mudstone/ash & ๑. Pleist & 1.58 & 66.3 & 2.84 & 69.5 & 2.05 & 850 & 280 & 120 & .570 & .730 & 4.1 & 0.6 & & & & & 14.3 & & & \\
\hline $671 B$ & $17 x$ & 151.5 & Calc mud & e. Pleistocene & 1.67 & 67 & 2.72 & 62.4 & 1.66 & 1003 & 600 & 530 & .403 & .473 & 9.4 & 0.72 & 44 & 3.3 & 42.8 & 53.7 & 1 & 1754 & 40.91 & 134 \\
\hline 6718 & $22 x-2$ & 199.1 & Calc. $n$ & e. Plets & 1.84 & 47.8 & .65 & 53.2 & 1.14 & 1365 & 520 & 480 & .845 & .885 & $1.2 \mathrm{E}-08$ & 0.3 & 7 & 0.7 & 29.1 & 70.2 & 19.9 & 1353 & & 103 \\
\hline 6710 & NAC-1 & 452.7 & Claystone & late Mlo & 1.65 & 69.3 & 2.52 & 60.9 & 1.56 & 3472 & & & & & & & 11 & 03 & 30.6 & 69.4 & 0.2 & 1284 & 46.18 & 82.6 \\
\hline $\begin{array}{l}6710 \\
6710\end{array}$ & $\begin{array}{l}\text { NAC-2 } \\
1 X-1-A\end{array}$ & $\begin{array}{l}454.1 \\
496.7\end{array}$ & $\begin{array}{l}\text { Claystone } \\
\text { Claystone }\end{array}$ & $\begin{array}{l}\text { late Miocene? } \\
\text { late Miocene? }\end{array}$ & $\begin{array}{l}1.75 \\
1.58\end{array}$ & $\begin{array}{l}58.9 \\
69.8\end{array}$ & $\begin{array}{l}2.67 \\
2.92\end{array}$ & $\begin{array}{r}58.4 \\
58.4 \\
\end{array}$ & $\begin{array}{l}1.41 \\
2.04\end{array}$ & $\begin{array}{l}3483 \\
3808\end{array}$ & 3000 & & .808 & & $6.46 \mathrm{E}-12^{\circ}$ & & 108 & 01 & 19.8 & 80.2 & $\begin{array}{l}0.3 \\
0.3\end{array}$ & $123=$ & 52.4 & 71 \\
\hline & $\begin{array}{l}\mathrm{V}=\text { =ert } \\
\mathrm{H}=\mathrm{hor} \\
\mathrm{T}=\text { triax }\end{array}$ & & 然 & tian this volume & & & & & & & & & & & & & & & & & & & & \\
\hline & & & & & & & & & REFER & RENCE & SITE & & & & & & & & & & & & & \\
\hline $\begin{array}{l}672 A \\
672 A\end{array}$ & $\begin{array}{l}2 \mathrm{H}-3-\mathrm{V} \\
2 \mathrm{H}-3-\mathrm{H}\end{array}$ & $\begin{array}{l}7.7 \\
7.8\end{array}$ & $\begin{array}{l}\text { Calc. mud } \\
\text { Calc. mud }\end{array}$ & $\begin{array}{l}\text { a. Pletstocenene } \\
\text { a. Plestistocene }\end{array}$ & $\begin{array}{l}1.6 \\
1.6\end{array}$ & $\begin{array}{l}90.3 \\
90.3\end{array}$ & 2.96 & $\begin{array}{l}69.8 \\
69.8\end{array}$ & $\begin{array}{l}2.32 \\
2.32\end{array}$ & $\begin{array}{l}362 \\
371\end{array}$ & $\begin{array}{l}200 \mathrm{~V} \\
130 \mathrm{H}\end{array}$ & $\begin{array}{l}175 \mathrm{~V} \\
100 \mathrm{H}\end{array}$ & $\begin{array}{l}184 \mathrm{~V} \\
93 \mathrm{H}\end{array}$ & $\begin{array}{l}139 \mathrm{~V} \\
43 \mathrm{H}\end{array}$ & $\begin{array}{l}2.65 \mathrm{E}-06 \\
7.62 \mathrm{E}-04\end{array}$ & $\begin{array}{l}0.85 \mathrm{~V} \\
0.47 \mathrm{H}\end{array}$ & $\begin{array}{l}19.5 \\
19.5\end{array}$ & $\begin{array}{l}20.13 \\
20.13\end{array}$ & $\begin{array}{l}34.9 \\
34.9\end{array}$ & $\begin{array}{l}43.5 \\
43.6\end{array}$ & $\begin{array}{l}35 \\
35\end{array}$ & $\begin{array}{l}87.4 \\
87.4\end{array}$ & $\begin{array}{l}31.4 \\
31.4\end{array}$ & $\begin{array}{l}56 \\
56\end{array}$ \\
\hline 672A & $5 \mathrm{H}-3$. & 36.24 & Calc. mud & Late Pllocene & 1.44 & 106 & 2.8 & 69 & 2.98 & 183 & $196 \mathrm{~T}$ & & 13 & & $3.74 \mathrm{E}-08$ & & 134 & & & & 48.6 & 142 & 42 & 100 \\
\hline 872A & $7 \mathrm{H}$. & 55.2 & Calc. & late & 1.61 & 77. & 27 & 67. & 2.04 & 300 & 310 & 20 & ic & -100 & & 0.8 & 43 & 21.35 & 53.2 & 24.9 & 27.6 & 116 & 34.7 & 81 \\
\hline (701 & 11 & 93.5 & Calc. $n$ & & & 56.6 & 2.65 & 69 & 2.31 & 540 & 34 & 30 & .20 & .24 & $9.33 \mathrm{E}-08$ & 0.6 & 93 & 3.4 & 28.2 & 68.3 & 41.5 & 113 & 32.2 & 80 \\
\hline & $11 \mathrm{H}-4-\mathrm{T}$ & 94.74 & Calc. mud & 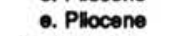 & 1.53 & 80 & & 68 & & 548 & $460 \mathrm{~T}$ & & -88 & & & & & & & & 31.7 & 132 & 60 & 72 \\
\hline 672A & $13 \mathrm{H}-3$ & 112.3 & Calc. mud & ate 1 & 1.68 & 68.9 & 2.6 & 62.7 & 1.68 & 669 & 380 & 260 & .289 & .409 & $1.07 E-07$ & 0.5 & 87.6 & 0.8 & 21.57 & 77.8 & 7.8 & 141 & 42.2 & ? \\
\hline & 18 & 16 & & 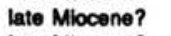 & 8 & 12 & & 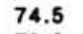 & 2. & 982 & & 53 & $-916 \mathrm{~V}$ & -92 & & & & 0.43 & 32.6 & 67.0 & 0 & 165 & 67.3 & 97 \\
\hline $72 A$ & $19 x-3-V$ & 165.9 & Calc. mud & late $M$ & 1.6 & 103 & 2. & 78 & 2.69 & 983 & $175 \mathrm{~V}$ & $140 \mathrm{~V}$ & $.808 \mathrm{~V}$ & $.843 \mathrm{~V}$ & $5.68 \mathrm{E}-08$ & $1.03 \mathrm{~V}$ & & 0.43 & 32.6 & 67.0 & 0 & 165 & 67.3 & 97 \\
\hline $672 A$ & $24 x-3$ & 213.3 & Claystone & 1. Oligocene & 1.72 & 60.9 & 2.7 & 59.8 & 1.49 & 1231 & 405 & 340 & .828 & .891 & $8.86 \mathrm{E}-0 \mathrm{~B}$ & 0.5 & 108 & 0 & 12.6 & 87.4 & 0.15 & 150 & 34.9 & 115 \\
\hline
\end{tabular}

MID-SLOPE SITE

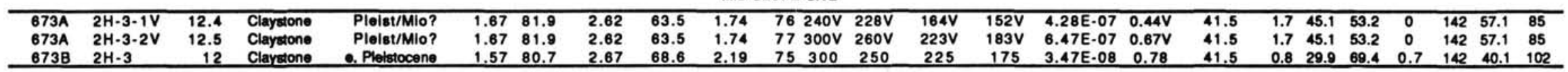

DEFORMATION FRONT SITE

\begin{tabular}{|c|c|c|c|c|c|c|c|c|c|c|c|c|c|c|c|c|c|c|c|c|c|c|c|}
\hline & & & & & & & & & & & & & & & & & & & & & & & \\
\hline $\begin{array}{l}678 A \\
676 A\end{array}$ & $\begin{array}{l}2 \mathrm{H} \cdot 3 \cdot \mathrm{T} \\
3 \mathrm{H}-3 \cdot \mathrm{V}\end{array}$ & $\begin{array}{r}10.64 \\
20.1\end{array}$ & $\begin{array}{l}\text { Calc. mud } \\
\text { Calc. mud }\end{array}$ & $\begin{array}{l}\text { a. Plestacene } \\
\text { a. Pleistocene }\end{array}$ & $\begin{array}{l}1.45 \\
1.62\end{array}$ & $\begin{array}{r}109 \\
73.4\end{array}$ & $\begin{array}{l}2.95 \\
2.85\end{array}$ & $\begin{array}{l}72.9 \\
67.4\end{array}$ & $\begin{array}{l}3.23 \\
2.04\end{array}$ & $\begin{array}{r}51 \\
107\end{array}$ & $\begin{array}{l}20 \mathrm{~T} \\
25 \mathrm{~V}\end{array}$ & $12 \mathrm{~V}$ & $\begin{array}{l}-31 \\
-82 V\end{array}$ & $.95 \mathrm{~V}$ & $3.21 \mathrm{E}-08$ & & $\begin{array}{c}72 \\
48.98\end{array}$ & & & $\begin{array}{l}30.1 \\
19.2\end{array}$ & 131 & 43 & 88 \\
\hline 676A & $3 \mathrm{H}-3 \cdot \mathrm{H}$ & 20.2 & Calc. mud & Plets: & 1.63 & 73.4 & 2.9 & 67.8 & 2.08 & 108 & $108 \mathrm{H}$ & $70 \mathrm{H}$ & 0 & $-38 \mathrm{H}$ & & & 48.98 & & & 19.2 & & & \\
\hline 678A & $5 \mathrm{H}-3$ & 39 & Calc. $m$ & Ple & 1.54 & 83 & 2.86 & 74.6 & 2.36 & 248 & $33 \mathrm{~T}$ & & .215 & & $1.06 \mathrm{E}-07$ & & 211 & & & 18.6 & 128 & 46 & 80 \\
\hline $\begin{array}{l}\text { 678A } \\
\text { 676A }\end{array}$ & $\begin{array}{l}6 \mathrm{H}-3 \cdot \mathrm{V} \\
6 \mathrm{H}-3 \cdot \mathrm{H}\end{array}$ & $\begin{array}{l}48.7 \\
48.8\end{array}$ & $\begin{array}{l}\text { Calc. mud } \\
\text { Calc. mud }\end{array}$ & $\begin{array}{l}\text { Pleisto } \\
\text { Plelsto }\end{array}$ & $\begin{array}{l}1.56 \\
1.56\end{array}$ & $\begin{array}{l}72.3 \\
72.3\end{array}$ & $\begin{array}{l}2.53 \\
2.51\end{array}$ & $\begin{array}{l}64.4 \\
64.2\end{array}$ & $\begin{array}{l}1.79 \\
1.77\end{array}$ & $\begin{array}{l}265 \\
266\end{array}$ & $\begin{array}{l}30 \mathrm{~V} \\
30 \mathrm{H}\end{array}$ & $\begin{array}{l}15 \mathrm{~V} \\
20 \mathrm{H}\end{array}$ & $\begin{array}{l}.235 \mathrm{~V} \\
.236 \mathrm{H}\end{array}$ & $\begin{array}{l}-250 \mathrm{~V} \\
-246 \mathrm{H}\end{array}$ & & & $\begin{array}{l}57.64 \\
57.64\end{array}$ & & & $\begin{array}{l}18.6 \\
18.6\end{array}$ & & & \\
\hline 676A & BH-3.T & 67.65 & Calc. mud & Iate & 1.68 & 57 & 2.84 & 60.5 & 1.62 & 390 & $52 \mathrm{~T}$ & & .338 & & $1.35 \mathrm{E}-08$ & & 205 & & & 48 & 84 & 33 & 51 \\
\hline 676A & $9 \mathrm{H}-3 . \mathrm{V}$ & 77.2 & Calc. mud & & 1.76 & 54.6 & 2.64 & 57.4 & 1.35 & 448 & $320 \mathrm{~V}$ & $285 \mathrm{~V}$ & $.128 \mathrm{~V}$ & $-163 \mathrm{~V}$ & $2.01 \mathrm{E} \cdot 07$ & & 108.63 & .7 & & 38.01 & 132 & 31.1 & 101 \\
\hline 676A & $9 \mathrm{H}-3$ & & Calc. m & & 1.7 & 54.6 & 2.64 & 57.4 & 1.35 & 449 & $310 \mathrm{H}$ & $250 \mathrm{H}$ & $-139 \mathrm{H}$ & $-199 \mathrm{H}$ & $1.52 \mathrm{E}-07$ & & 108.6 & & & 38.01 & 132 & 31.1 & 101 \\
\hline 676A & $11 \mathrm{H}-4-\mathrm{T}$ & 97.63 & Calc. mud & & 1.74 & 48 & & 57.5 & & 592 & $400 \mathrm{~T}$ & & .192 & & & & & & & 30.9 & $\pi$ & 13 & 64 \\
\hline $676 \mathrm{~A}$ & $12 X-3 \cdot V$ & 105.6 & Calc. mud & late Pliocene & 1.78 & 48.2 & 2. & 55.4 & 1.24 & 647 & $420 \mathrm{~V}$ & $350 \mathrm{~V}$ & $-227 V$ & $.297 V$ & & & & 22.7 & 75.1 & 22.23 & 98.5 & 29.8 & 68.7 \\
\hline 676A & $12 X \cdot 3 \cdot H$ & 105.7 & Calc. mud & late Pllocene & 1.78 & 48.2 & 2.7 & 55.4 & 1.24 & 648 & $360 \mathrm{H}$ & $300 \mathrm{H}$ & $.288 \mathrm{~V}$ & $-348 \mathrm{H}$ & $6.14 \mathrm{E} \cdot 08$ & $.44 \mathrm{H}$ & 123.22 & $2.2 \quad 22.7$ & 75.1 & 22.23 & 98.5 & 29.8 & 68.7 \\
\hline
\end{tabular}



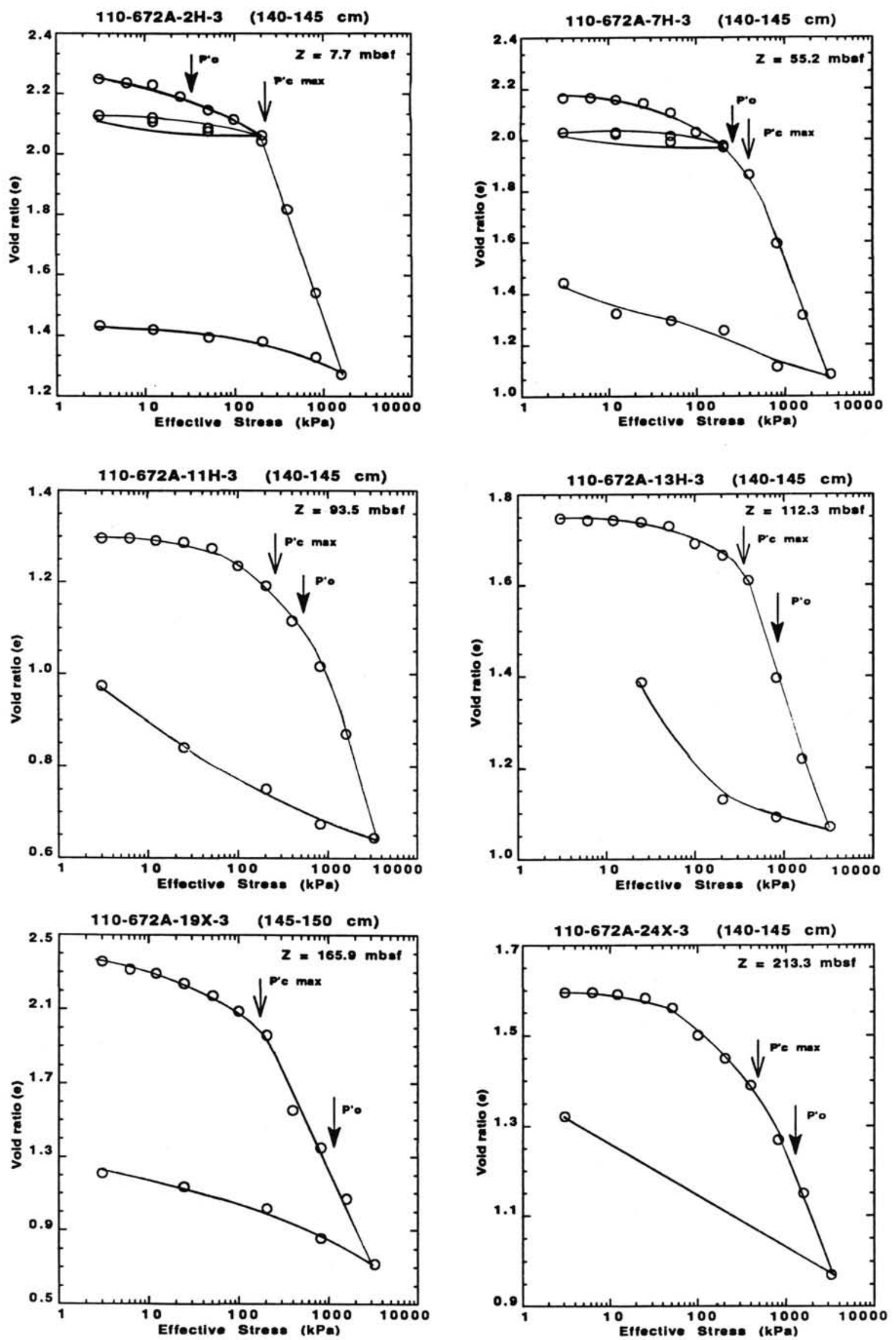

Figure 2. Void ratio vs. effective stress curves for Site 672 consolidation samples. Estimated pre-consolidation $\left(P_{c}^{\prime}\right)$ and effective overburden $\left(P_{o}^{\prime}\right)$ stresses are shown. 


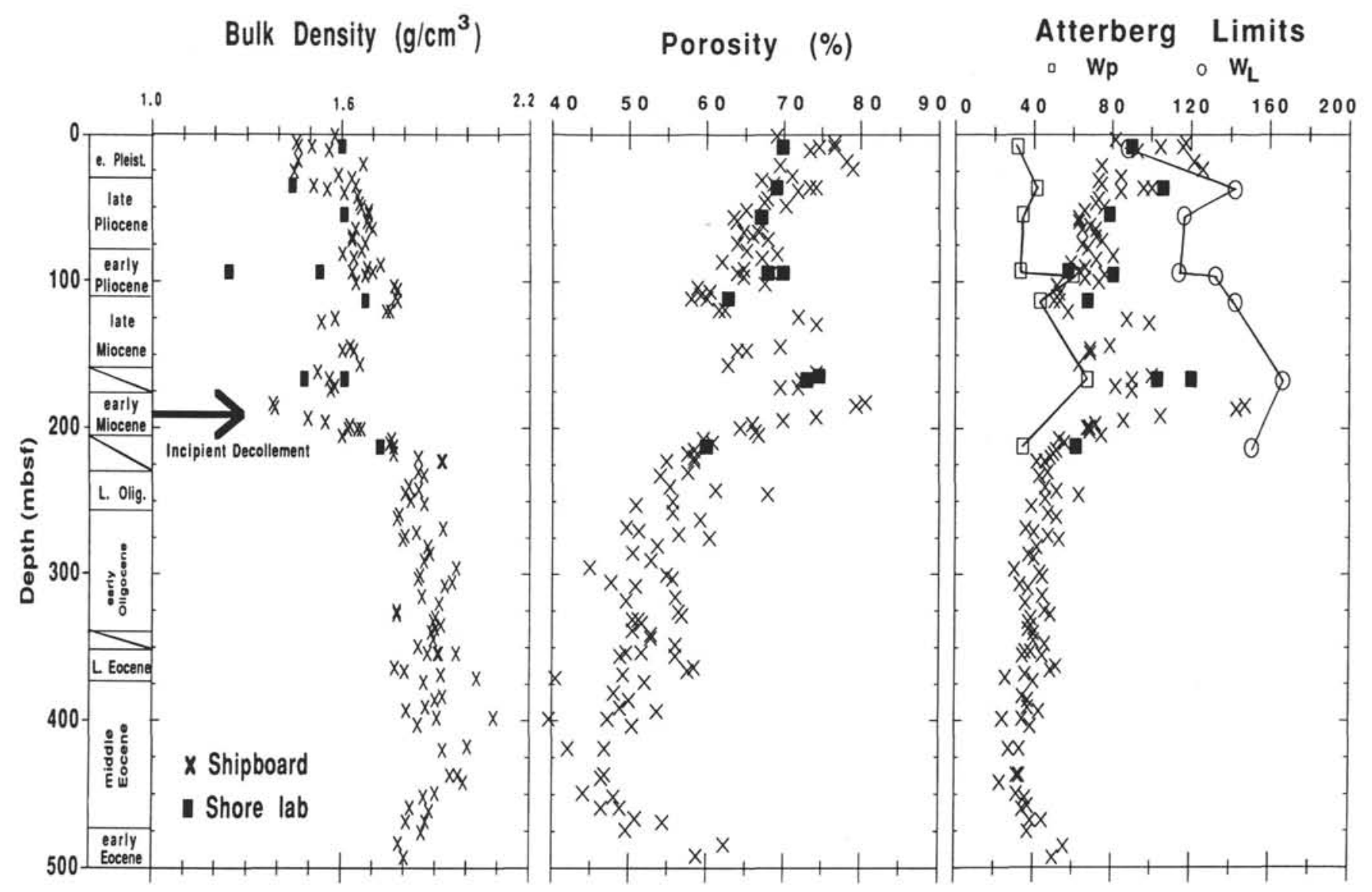

Figure 3. Summary of physical properties vs. depth for Site 672 sediments.

is slightly more overconsolidated $(\mathrm{OCD}=+18$ to +53$)$ than the surface sample. Just as at Site 672, both of these samples are of early Pleistocene age, as is Sample 110-671B-3H-4 (140-150 $\mathrm{cm})$, which is underconsolidated at a depth of 22.9 mbsf. Sample 110-671B-9H-2 (140-150 cm), tested in both directions at $76.8 \mathrm{mbsf}$, is also slightly underconsolidated vertically; both Samples 110-671B-3H-4 and -9H-2 (vertical) have OCDs of about -65 to -75 and -118 , respectively. Generally, the OCD values in the upper $80 \mathrm{~m}$ of Site 671 proceed from slightly overconsolidated to progressively underconsolidated below this depth. The behavior difference between samples at 21.3 and 22.9 mbsf may reflect local sedimentological fabric differences. Close observation of the shallower sample reveals small (1-2 mm) more indurated mud within a softer matrix, possibly indicating local sediment remobilization. The greatest OCD value of -835 was obtained for Sample 110-671B-22X-2 (140-150 cm), at $199.1 \mathrm{mbsf}$.

Samples below 200 mbsf were not considered of adequate quality for consolidation testing due to their stiffness and apparent disturbance. However, three samples from between 450 and 500 mbsf were tested. The upper two samples complement the data from higher in the section, exhibiting increasing states of underconsolidation with depth. However, the sample from immediately above the décollement appears overconsolidated, and lies within the upper Miocene(?) mudstone immediately overlying the décollement (Fig. 8). This clear shift in consolidation behavior reflects local sediment deformation and concurrent dewatering into permeable scaly mudstone of the décollement.
In-situ intrinsic permeability for Site 671 samples ranges from $10^{-6}$ to $10^{-11} \mathrm{~cm} / \mathrm{s}$ (Table 1 and Fig. 8). These permeabilities appear to decrease monotonically with increasing depth, as expected from progressive consolidation. Sample 110-671B-9H-2 was also tested in the vertical $(140-145 \mathrm{~cm})$ and horizontal $(145-$ $150 \mathrm{~cm}$ ) directions for permeability anisotropy. Results indicate that differences between vertical and horizontal permeability are variable and less than one order of magnitude. The mudstone at $497 \mathrm{mbsf}$ has a very low permeability $\left(10^{-11} \mathrm{~cm} / \mathrm{s}\right)$, which may account for the apparent "cap" overlying the décollement.

The physical properties at Site 671 generally exhibit a normal response to burial punctuated by thrust faults and lithologic changes (Fig. 8). Structural disruptions of the nearly regular downhole trend in physical properties occur at 128 mbsf (thrust fault A) and 370 mbsf (thrust fault B). The upper thrust fault emplaced upper Miocene over lower Pleistocene sediments, registering as an abrupt porosity increase from above to below this fault surface. Thrust fault B marks a contact between calcareous mud(stone) of lithologic Unit I from the mud(stone) facies of lithologic Unit II.

Unit II sediments exhibit higher porosities than those with a calcareous component, providing an example of the lithologic control on physical properties. High porosities at Site 671 are also associated with smectitic and radiolarian mudstones occurring above and within the décollement. The interesting aspect of this latter section is the pronounced porosity decrease that occurs within the same lithologic unit. This abrupt downhole porosity 


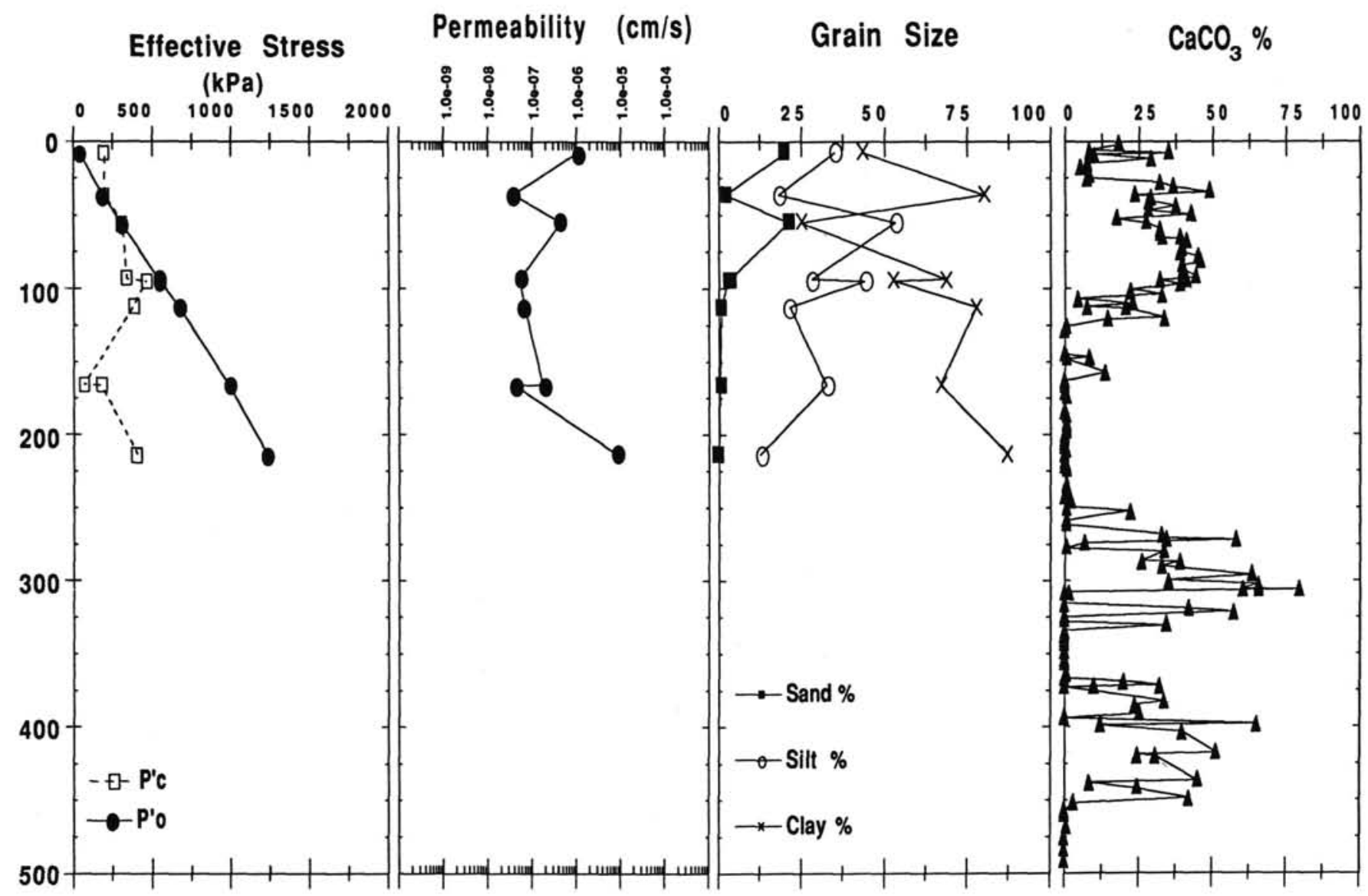

Figure 3 (continued).

loss is tied to structural shearing of fabric and short drainage paths within that interval (Shipboard Scientific Party, 1988 and Moore, Mascle, et al., 1988).

\section{Site 673}

Two holes were drilled at Site $673,8 \mathrm{~km}$ west and upslope of Site 671 . Hole $673 \mathrm{~A}$ cored $36 \mathrm{~m}$ and Hole $673 \mathrm{~B}$ cored $331 \mathrm{~m}$ of hemipelagic Pleistocene-Miocene yellowish brown to olive-gray calcareous mud and marl, reworked claystone blocks, and matrix-supported conglomerates and breccias. Well-documented biostratigraphic inversions and a 50-m-thick overturned section show large-scale folding and distinguish Site 673 from the sites closer to the deformation front. Two samples were obtained from Holes 673A and 673B from 12.4 and $12 \mathrm{mbsf}$, and three vertical consolidation tests were performed on these samples (Fig. 9). The three samples are overconsolidated, with OCD values between +170 and +225 . The samples have relatively low porosity (Fig. 10), void ratio, and permeability for shallow sediments when compared to similar sediments at Sites 671 and 676 (Wilkens et al., this volume) and vane shear strength is also high. Although it is tempting to view the Site 673 shore-based consolidation data as more evidence of lateral strengthening due to progressive deformation in the accretionary complex, the samples tested are actually from within a slope-slump deposit that has a stress history which is distinct from the accretion-subduction process. Consequently, deeper samples are required to estimate the stress state at this site.

\section{DISCUSSION}

\section{Consolidation}

The process of sediment consolidation for most marine sediments is a result of gravitational loading from the weight of overlying material. Over time the overburden causes void space to decrease and sediment particles to become more closely arranged as the pore fluids are expelled. Very low permeability in fine-grained marine sediments may hinder the process of dewatering during rapid vertical or tectonic loading events. This hinderance may result in excess pressures in these pore fluids (Taylor and Bryant, 1985). Within active margins, lateral tectonic compression adds a horizontal component to vertical loading. The application of these combined stresses may be transferred to the pore fluid, resulting in the generation of excess pore pressures. Excess pore pressures, in turn, retard the sediment response to higher applied stress because the effective stress results from the difference between total stress and pore pressure. The net result therefore may be a sediment that locally has not increased in shear strength despite the increase of applied stress.

An initial goal of this research was to compare the relative degrees of consolidation in the different sediment columns cored across and in front of the lower slope of the Barbados Ridge, and to determine if, and how much, excess pore pressure exists within the forearc. Sediment consolidation is therefore approached from two perspectives: (1) the physical evidence; and (2) the mechanical evidence. 


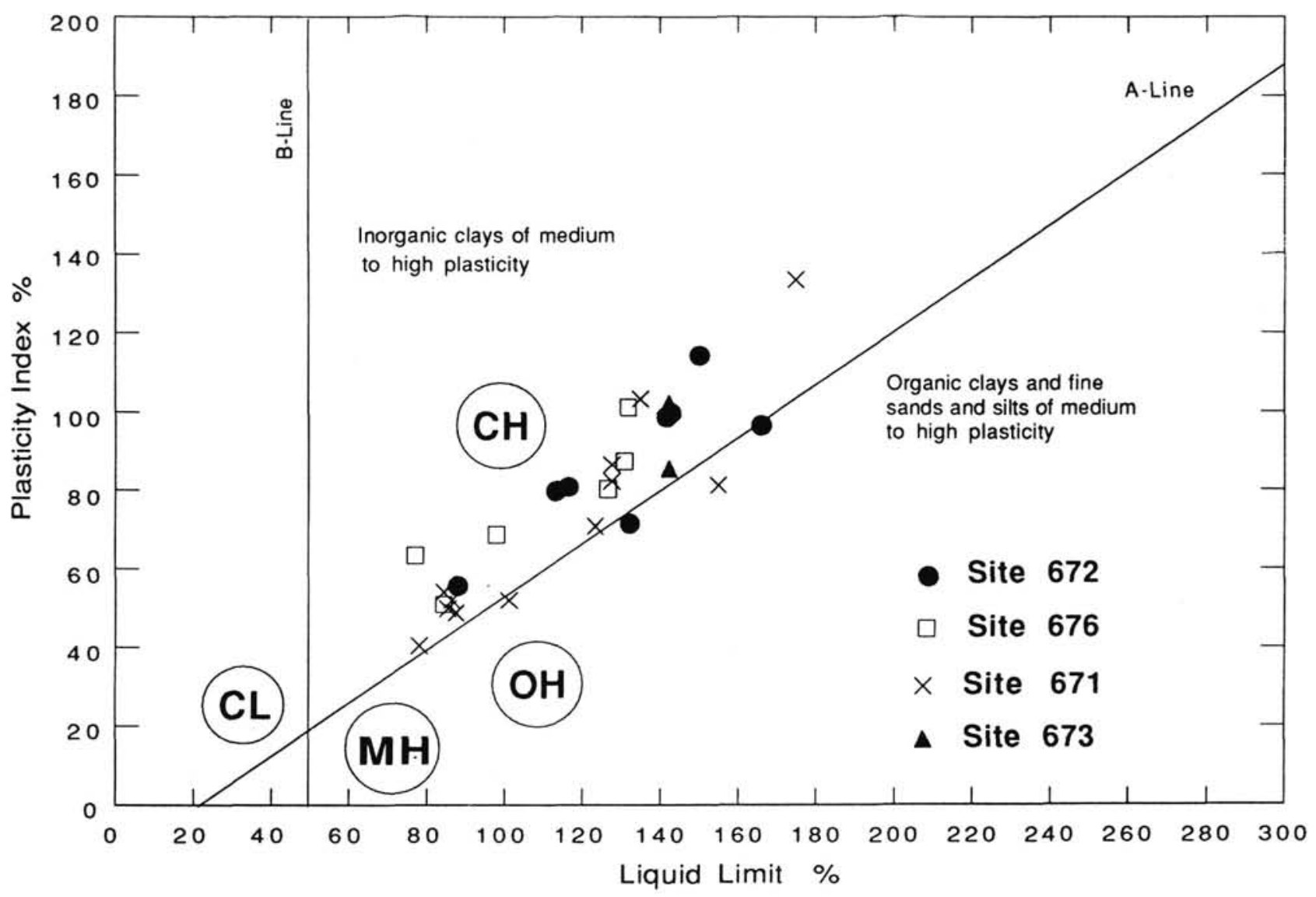

Figure 4. Atterberg limits classification chart for Leg 110 samples. Note that nearly all samples correspond to inorganic clays of medium to high plasticity. Letters refer to the United Soils Classification System (Lambe and Whitman, 1969).

Shipboard Scientific Party (1988) presented a summary of porosity changes in Pliocene sediment from similar depths at all sites on the lower Barbados forearc. The porosity reduction in that sediment clearly indicates that, despite similar burial depths, sediment within the accreted wedge has undergone additional consolidation. This is further verified by subsequent shore-based analyses of porosity data (Wilkens et al., this volume), and void ratios corrected for sediment rebound (Fig. 11). The measured vane shear strengths at the Leg 110 sites also indicate an apparent lateral strengthening of sediments. The linear regression of shear strength vs. depth measured at Sites 671, 672, and 676 are:

$$
\begin{array}{ll}
\text { Site } 671 & S_{u}=8.6+(1.51) Z \\
\text { Site } 676 & S_{u}=34+(0.82) Z \\
\text { Site } 672 & S_{u}=16.5+(0.70) Z
\end{array}
$$

where $S_{u}$ is the undrained vane shear strength $(\mathrm{kPa})$ and $Z$ is depth (mbsf). These relations show a consistent increase in strength vs. depth and across the arc, where the gradient at Site 671 is over twice that of Site 672 .

A comparison of permeabilities estimated at in-situ conditions also reveals a systematic decrease across the arc at similar burial depths. The total permeability range for sediments at common depths is only about one order of magnitude (Figs. 3, 6 , and 8). With the exception of $k_{c}$ values, the physical evidence of lateral consolidation appears to be substantiated, although the changes are relatively subtle. The sediment deformation seemingly resulting from lateral compression within the accreted section is taking place with only modest dewatering and volume loss.

The second aspect of sediment consolidation, the mechanical evidence, is derived from the laboratory testing described above. The apparent overconsolidation in Leg 110 samples obtained from near the mudline has been commonly noted in the upper 10-15 mbsf for most marine sediments. This overconsolidated behavior is attributed to origin cohesion (Skempton, 1970; Bryant et al., 1981; Marlow et al., 1984), a result of the physicochemical internal attraction bonding the sediment particles to each other and which is greater than the overburden stress applied to them at shallow sub-bottom depths. However, another common cause of overconsolidation is the removal of overburden due to erosion or other means. Given the lower Pleistocene age of the sediments at the seafloor, the possibility that several meters of Holocene-upper Pleistocene sequence has been removed from this area by western boundary bottom currents, or by slumping off the Tiburon Rise, or off the accretionary complex itself, cannot be discounted. We stress, however, that the overconsolidation measured in near-surface samples is subtle relative to the degree of underconsolidation observed at greater depths.

Sediments from Sites 672,676 , and 671 are progressively underconsolidated with increasing depth below the seafloor (Fig. 12). At Site 672 , sediments depart from a near-normal consolidation profile below 55 mbsf, with maximum underconsolidation occurring at $165 \mathrm{mbsf}$, in sediments at the top of the eastward-propagating décollement. Results from Site 543 indicate OCD values of nearly -150 and -300 at 112 and 160 mbsf, respectively (Marlow et al., 1984). These two samples are from the 

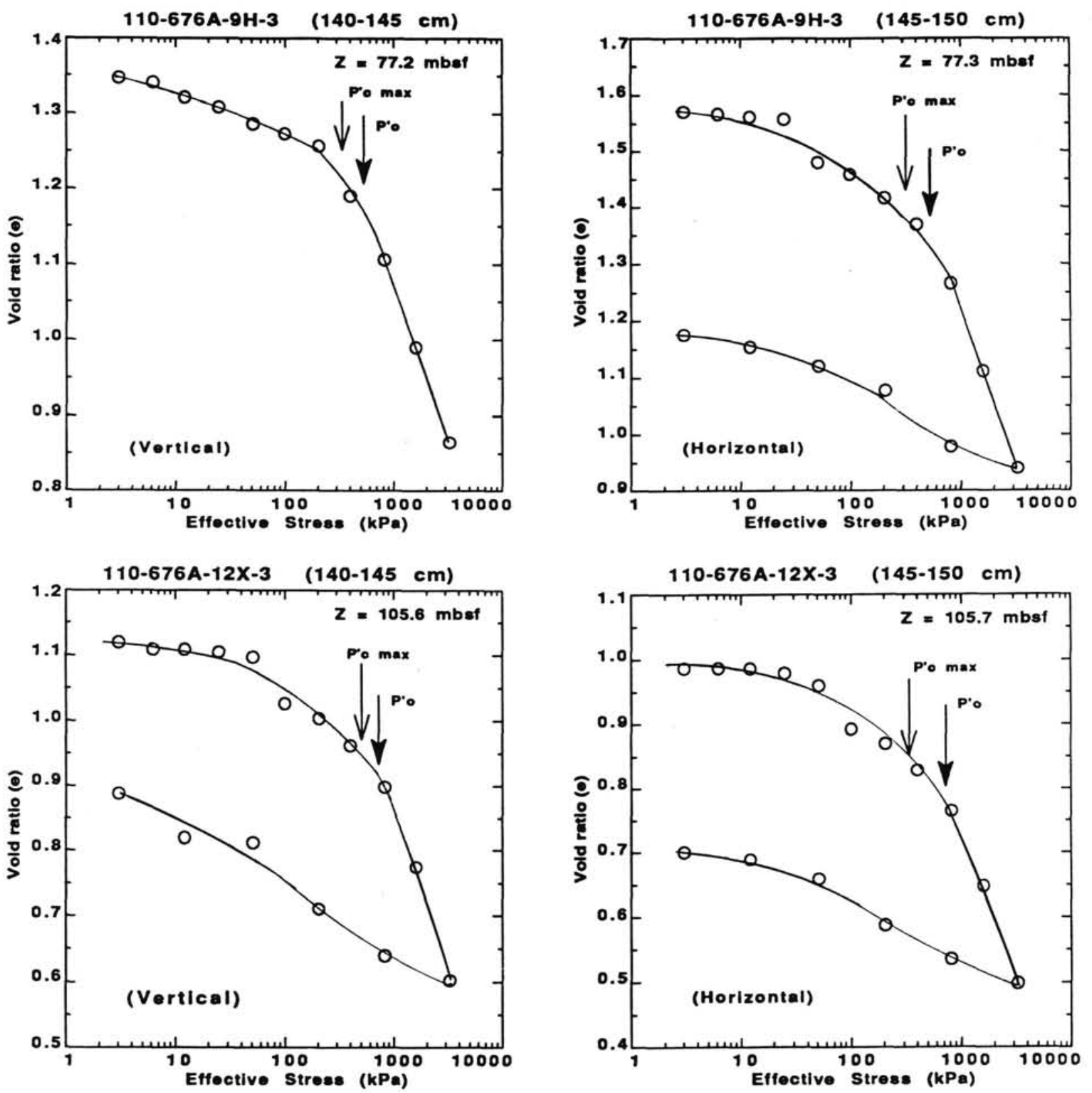

Figure 5. Void ratio vs. effective stress curves for Site 676 consolidation samples.

mud(stone) section overlying the radiolarian mudstone interval at this reference site, located $3.5 \mathrm{~km}$ east of the deformation front but $22 \mathrm{~km}$ north of the other sites. Site 676 samples are all underconsolidated within the upper 106-m interval tested. These samples are limited to the lower Pleistocene-upper Pliocene section and hence can only be compared with the upper two samples from Site 672. This comparison, however, shows that these accreted sediments at the leading edge of the forearc are more underconsolidated than the incoming equivalent lithostratigraphic section. Furthermore, this underconsolidation occurs despite a thickening of the section through stratigraphic repetition tied to the thrust fault at $35 \mathrm{mbsf}$. Indeed, this thickening may represent rapid vertical tectonic loading of the underlying sediment, leading to additional excess pore pressure.

Site 671 sediments appear to be nearly normally- to overconsolidated to about 21 mbsf, below which their underconsolidated state is increasingly accentuated. This pattern of under- consolidation is consistent with the test results of Marlow et al. (1984) for Site 541, which is located less than a kilometer southeast of Site 671 . The DSDP Leg 78A samples are also nearly normally consolidated to $55 \mathrm{mbsf}$, below which the degree of underconsolidation increases to an OCD close to -400 at about $130 \mathrm{mbsf}$, the same as Site 671 . Immediately overlying the décollement, however, mudstones appear to be overconsolidated. A short dewatering path to the fractured permeable paths within the décollement for these sediments may account for the apparent change in consolidation behavior.

The two different aspects of consolidation, mechanical and physical, appear to contradict each other. Where physical aspects of the accreted sediments indicate volume reduction, loss of permeability, and strengthening, the mechanical aspects suggest progressive underconsolidation across the prism. A possible reconciliation between these two conflicting aspects of convergence may lie in the true directions of the in-situ principal 


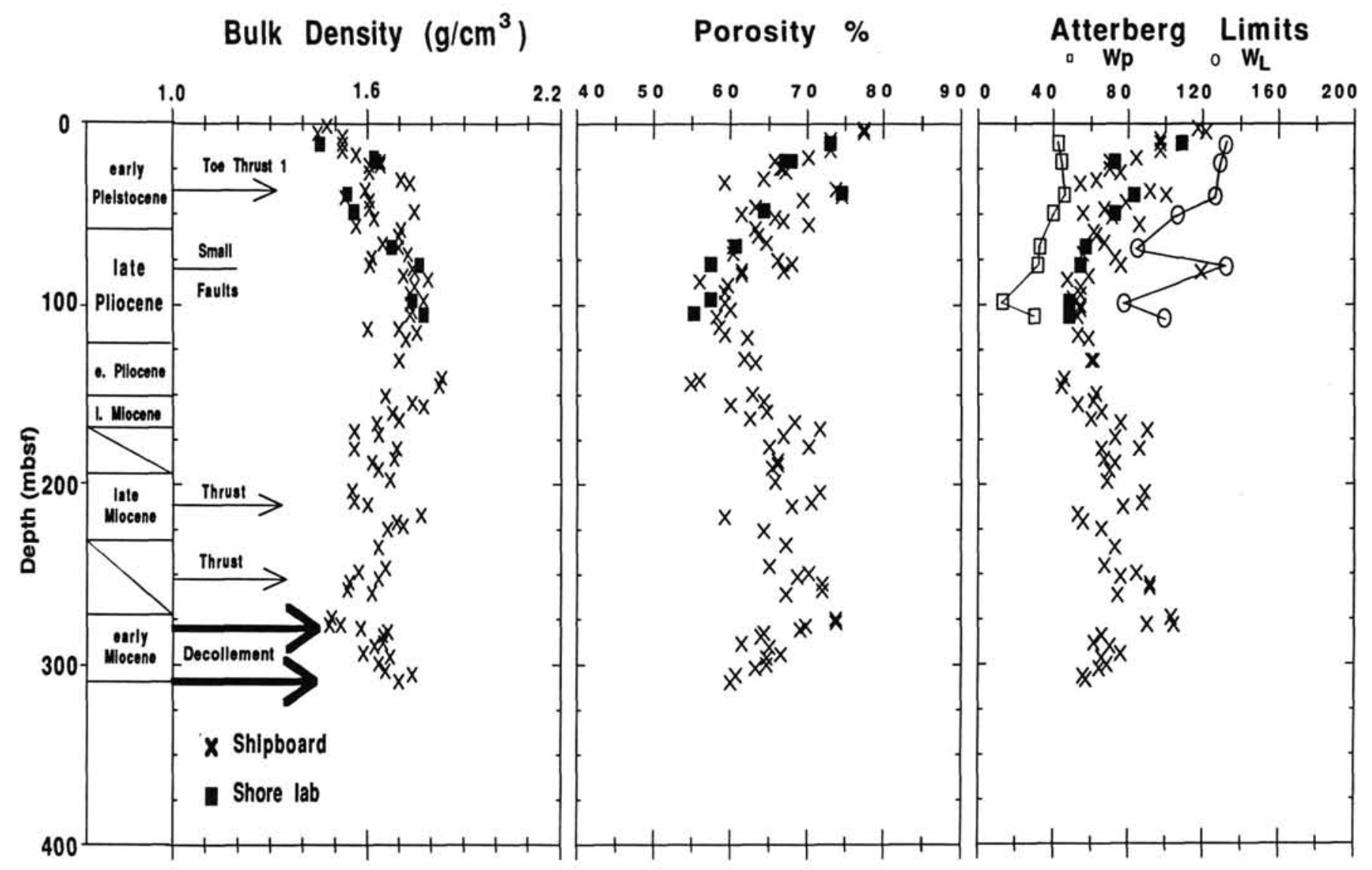

Figure 6. Summary of physical properties vs. depth for Site 676 sediments.

stress vectors across the arc. Laboratory consolidation tests by oedometer presume vertical overburden is the maximum principal stress direction. However, borehole instability on Leg 78A (Davis, 1984) and triaxial tests performed on Leg 110 core samples (Moran and Christian, this volume) indicate that the maximum compressive stress direction in these accreted sediments is most likely not vertical.

In addition to low permeability, underconsolidated conditions may also arise from high sedimentation rates. Excess pore pressures may approach lithostatic values during rapid accumulation of fine-grained sediments. Bredehoft and Hanshaw (1968) theoretically demonstrated this relationship for sediments accumulating at rates of $500 \mathrm{~m} / \mathrm{m}$.y., assuming permeabilities of less than $1 \times 10^{-8} \mathrm{~cm} / \mathrm{s}$, a value which is in the range of the Leg 110 samples. Marlow, et al. (1984) used the method of Gibson (1958) for analysis of underconsolidation resulting from rapid sedimentation and concluded that the observed underconsolidation in this area is not induced by the sedimentation rate, a conclusion further substantiated by the Leg 110 results.

\section{Permeability}

The permeability of a sediment is a parameter that is interdependent with the other physical properties affecting the consolidation process and is dependent upon the in-situ stress field. The rate of consolidation is controlled by how fast the pore-water can dissipate under a given pressure gradient. In marine settings, the factors that principally influence this consolidation process are depth of burial, grain size, porosity, and permeability (Taylor and Bryant, 1985; Bryant et al., 1986b). Porosity is a measure of the volume of voids in the sediment, and permeability depends on the interconnection (tortuosity) of these voids to achieve fluid flow through the sediment. Larger and more numerous voids are found in well-sorted material composed of larger grains, but void spaces decrease due to the overburden load at greater depths of burial, and smaller grains can fill the decreasing void spaces, hindering permeability and therefore the dewatering process necessary for normal consolidation. Low permeability therefore directly contributes to excess pore pressures by restricting flow as the void space decreases. The result may be an underconsolidated sediment in which the fine-grained matrix is partially supported by the pore fluid.

The Leg 110 sediments exhibit characteristic permeabilities of fine-grained marine sediments. The calcareous component in these lithologies appears to influence the void ratio-permeability relationship (Fig. 13). Excluding the two tests for the sandy sample from Site 672 with abnormally high permeabilities, the decrease in calcareous component in sediments of nearly equivalent void ratio corresponds to a decrease in permeability. Bryant et al. (1981) discussed the general permeability characteristics for oceanic sediments of different grain sizes and compositions. Their permeability range for silty clays to clayey silts overlaps neatly with the Leg 110 measured permeabilities. The range of $10^{-7}$ to $10^{-11} \mathrm{~cm} / \mathrm{s}$ for Leg 110 values, a measure of intergranular permeability, provides limits for hydrologic modeling. In general, vertical vs. horizontal permeability measurements at all sites give mixed results, indicating that the existence of anisotropy in the tested sediments is most likely a function of very localized conditions.

Fluid flow through scaly clays within the décollement, within vein networks in accreted sediments, along faults, and in coarsergrained horizons, is apparent in mineral precipitation and geochemical signatures in pore fluids (Shipboard Scientific Party, 


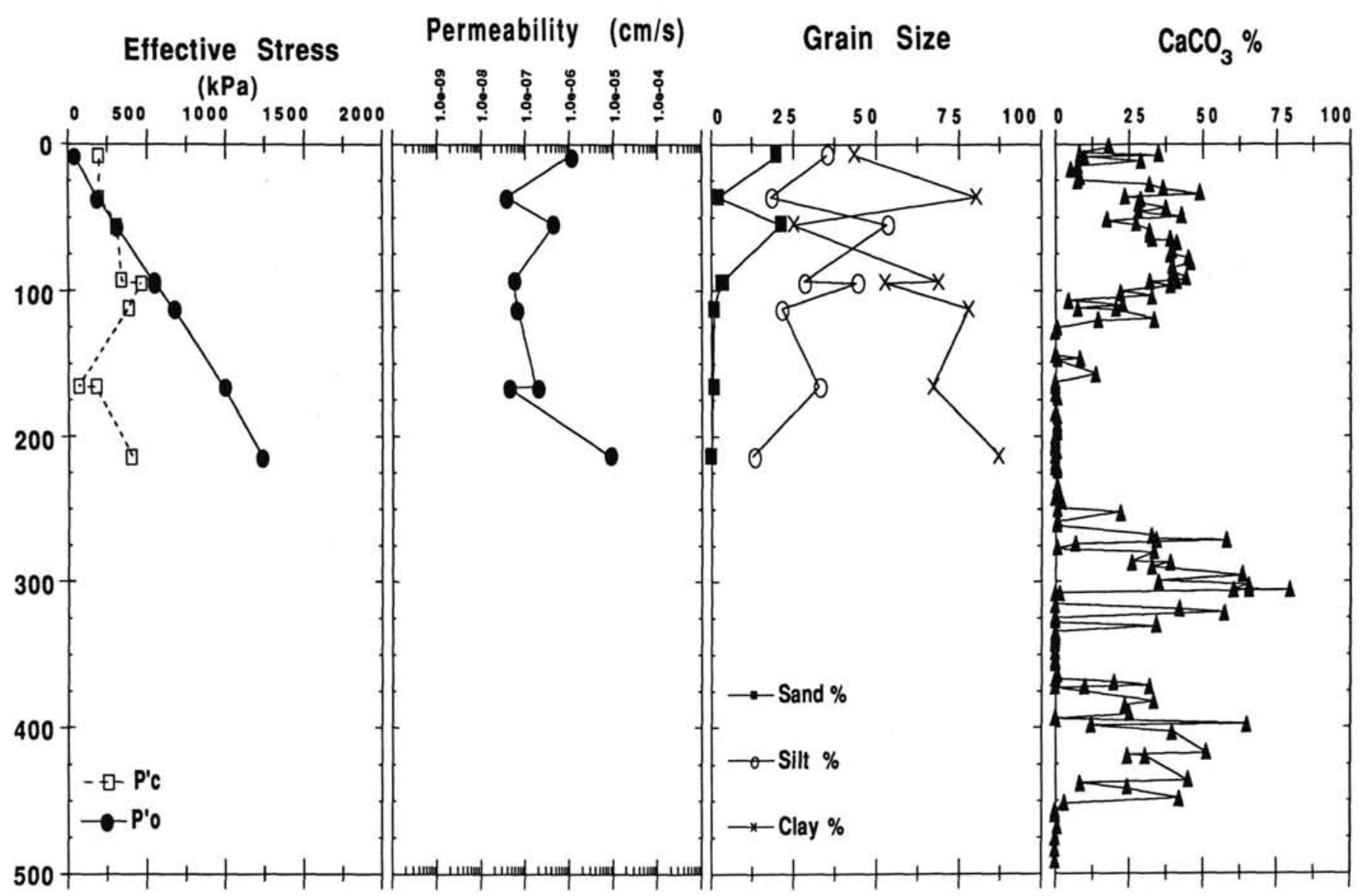

Figure 6 (continued).

1988; Gieskes et al, this volume). The larger scale formation permeability associated with structural features in the forearc could not be measured, nor do we know how this more permeable system may operate on a temporal and spatial basis. From Leg 78A geochemical data, Gieskes et al. (1984) concluded that if advection of interstitial fluids is occurring associated with the subducting sediment slab, this advection cannot be in an upward direction, and fluids must be flowing laterally. The low permeability at Site 671 (500 mbsf) may represent a cap to fluid flux through mudstones overlying the décollement. This cap impedes upward fluid advection of incompressible pore-water contained in fine-grained, low-permeability, subducting sediments. This process creates a high pore-pressure, low-strength zone as the sediment is brought beneath the deformation front and subjected to a rapidly increasing stress field resulting in "lubricated subduction" (Marlow et al., 1984). This plane of weakness facilitates further sediment subduction and lateral advection of fluids, helping propagate the incipient décollement as far as 6 $\mathrm{km}$ to the east of the deformation front. However, physical evidence of compaction and geochemical pore-fluid characteristics indicate that farther west in the zone of accretion and deformation, upward flow of fluids is occurring via narrow fault planes and other vertical pathways.

Because offscraped Leg 110 sediments have a very low intergranular permeability, if we apply vertical and lateral loads to the section, with only limited dewatering, the in-situ pore pressures will increase, limiting a net increase in effective stress and hence consolidation. Should this process continue to develop, the low strength of the sediment will allow it to fail under increasing applied stress. This failure, in the form of a propagat- ing fracture, scaliness, or veining provides a conduit for fluid escape, decreasing the pore pressure from the zone of failure and allowing some consolidation to take place. The combination of intergranular and fracture permeability, operating on variable time scales, together regulate the deformation and dewatering of accreted and subducted sediments (Moore, Mascle, et al., 1988). Carson and Berglund (1986) modeled similar effects of limited fluid flow, fracture development, and subsequent dewatering in a laboratory simulation of the convergence process.

\section{Pore Pressures}

The state of effective stress within the sediment column is a function of the total stress environment and pore pressure. Any models of sediment accretion, faulting, and subduction must either make measurements of pore-pressure distribution or infer them. The shipboard efforts to obtain in-situ pore-pressure information from the drill-in packer unfortunately were unsuccessful (Mascle, Moore, et al., 1988). However, an estimate of the pore-pressure conditions may be inferred from the relative degree of consolidation, assuming that pore pressures in excess of hydrostatic account for the underconsolidated state measured in oedometer tests.

The maximum expected pore pressures from consolidation tests are plotted in Fig. 14. These values, obtained from the maximum OCD, indicate a substantial pore-pressure build-up in sediments across the forearc. The distribution of pore-pressure with depth in the sediment section does not appear to be affected by occurrence of major thrust faults, although much denser sampling would be required to test this idea. Geochemi- 

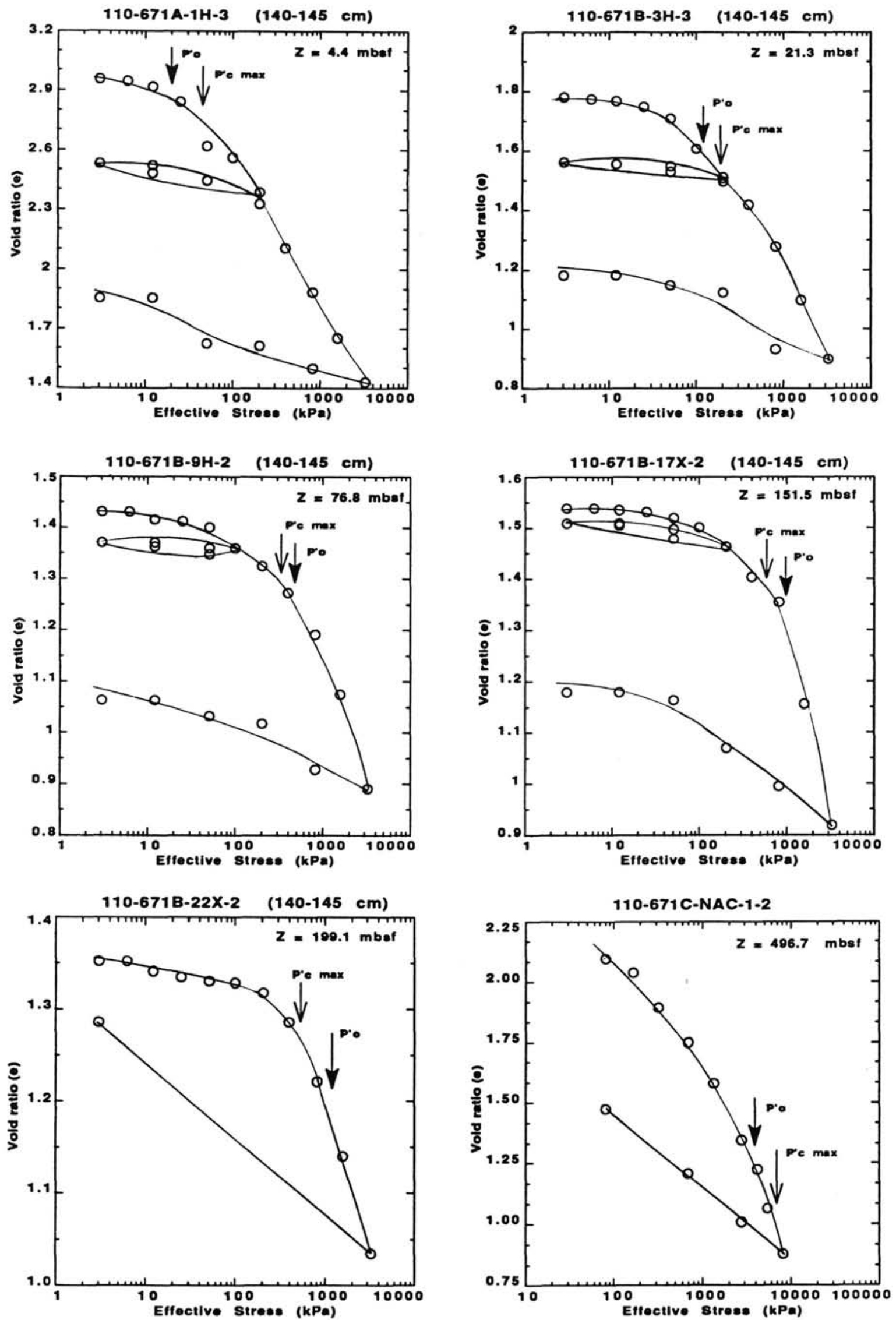

Figure 7. Void ratio vs. effective stress curves for Site 671 consolidation samples. 
cal signatures of pore fluids near the thrust faults suggest that only local dewatering is occurring, and hence sustained overpressures within tectonics packages are quite feasible. Maximum pore pressures of $2500 \mathrm{kPa}$, at Site 671 , are nearly two thirds of the overburden load at those depths. This estimate is surprisingly close to the pore pressure infered from the "inadvertant packer experiment" of DSDP Leg 78A (Biju-Duval, Moore, et al., 1984). Pore pressures near $900 \mathrm{kPa}$ at reference Site 672 , immediately above the propagating décollement, are in fact almost equivalent to the estimated overburden.

\section{CONCLUSIONS}

A full understanding of the stresses, pore pressures, and permeabilities controlling sediment accretion, development of the décollement and associated thrust faults, and the process of subduction really requires in-situ measurements. However, our insight into the complexities of trench-forearc geology is greatly improved by the numerous physical and mechanical tests performed on cored samples already recovered. Test results from this study indicate that in the Barbados Ridge area:

1. Sediments are almost exclusively underconsolidated, and show a greater degree of underconsolidation with depth below the seafloor. This progressively underconsolidated aspect may reflect excess pore pressures resulting from vertical tectonic loading compounded by rotation of the principal stress vector from a vertical to sub-horizontal direction.

2. Lateral compression and dewatering by fracture permeability is taking place in accreted sediments, resulting in very limited pore-volume reduction and sediment strengthening.

3. Intergranular permeabilities vary across the forearc and within lithostratigraphic horizons. These permeabilities range from $10^{-6}$ to $10^{-11} \mathrm{~cm} / \mathrm{s}$ and serve as limits for geohydrologic modeling.

4. Estimates of maximum pore pressures indicate a build-up of pore pressure in the thrust packages and at the reference site. Excess pore pressures nearly equivalent to the estimated overburden stress appear to exist immediately overlying the incipient décollement at reference Site 672 .

\section{ACKNOWLEDGMENTS}

The authors acknowledge the shipboard assistance provided by the stupendous ODP technicians and fellow scientists, both Teams A and B. We are also grateful for the skilled leadership provided by our departed friend, Lamar Hayes. The manuscript has benefited greatly from the comments offered by Kate Moran, Harold Christian, and Mark Brandon. Technical advice and support provided by Bill Bryant, Alan Pittenger, Bryan Trimm, and John Hedrick is much appreciated. Support was provided by a JOI/NSF(USSAC) grant to E.T. This research is a partial fulfillment of a M.Sc. thesis by J. L.

\section{REFERENCES}

Biju-Duval, B., Moore, J. C., et al., 1984. Init. Repts. DSDP, 78A: Washington, (U.S. Govt. Printing Office).

Bowles, J. E., 1978. Engineering Properties of Soils and Their Measurements: New York (McGraw-Hill).

Boyce, R. E., 1976. Definitions and laboratory techniques of compressional sound velocity parameters and wet-water content, wet-bulk density and porosity parameters by gravimetric and gamma ray attenuation techniques. In Schlanger, S. O., Jackson, E. D., et al., Init. Repts. DSDP, 33: Washington (U.S. Govt. Printing Office), 931-958.

Bredehoeft, J. D., and Hanshaw, B. B., 1968. On the maintenance of anomalous fluid pressures: I. Thick sedimentary sequences. II. Source layer at depth. Geol. Soc. Am. Bull., 79:1097-1122.

Bryant, W. R., Bennett, R. H., and Katherman, C. E., 1981. Shear strength, consolidation, porosity, and permeability of oceanic sedi- ments. In Emiliani, C. (Ed.), The Sea (Vol. 7): New York (Wiley), 1555-1616.

Bryant, W. R., Wetzel, A., Taylor, E., and Sweet, W., 1986a. Consolidation characteristics and permeability of Mississippi fan sediments. In Bouma, A. H., Coleman, J. M., et al., Init. Repts. DSDP, 96: Washington (U.S. Govt. Printing Office), 797-809.

Bryant, W. R., Wetzel, A., and Sweet, W., 1986b. Geotechnical properties of intraslope basin sediments, Gulf of Mexico, Deep Sea Drilling Project Leg 96, Site 619. In Bouma, A. H., Coleman, J. M., et al., Init. Repts. DSDP, 96: Washington (U.S. Govt. Printing Office), 819-824.

Carson, B., and Berglund, P. L., 1986. Sediment deformation and dewatering under horizontal compression: experimental results. In Moore, J. C., (Ed.), Structural Fabrics Preserved in Deep Sea Drilling Project Cores From Forearcs. Mem. Geol. Soc. Am., 166:135150 .

Casagrande, A., 1936. The determination of the preconsolidation load and its practical significance. Proc. First Inter. Conf. Soil Mech. and Found. Eng., Cambridge, 60-64.

Davis, D. M., 1984. The compressive mechanics of accretionary wedges applied to the Leg 78A study area near Barbados. In Biju-Duval, B., Moore, J. C., et al., Init. Repts. DSDP, 78A: Washington, (U.S. Govt. Printing Office), 569-579.

Davis, D. M., and von Huene, R., 1987. Inferences on sediment strength and fault friction from structures at the Aleutian Trench. Geology, $15: 517-522$

Folk, R. L., 1974. Petrology of Sedimentary Rocks: Austin (Hemphill Publ. Co.).

Gibson, R. E., 1958. The progress of consolidation in a clay layer increasing in thickness with time. Geotechnique, 8:71-182.

Gieskes, J. M., Elderfield, H., Lawrence, J. R., and LaKind, J., 1984. Interstitial Water Studies, Leg 78A. In Biju-Duval, B., Moore, J. C., et al., Init. Repts. DSDP, 78A: Washington, (U.S. Govt. Printing Office), 377-384.

Head, K. H., 1982. Manual of Soil Laboratory Testing, Volume 2: Permeability, Shear Strength and Compressibility Tests: New York (Halstead Press/Wiley).

Lambe, T. W. 1951. Soil Testing for Engineers: New York (Wiley).

Lambe, T. W., and Whitman, R. V., 1969. Soil Mechanics: New York (Wiley).

Lowe, J., Zaccheo, P. F., and Feldman, H. S., 1964. Consolidation testing with back pressure. J. Geotech. Eng. Div., Am. Soc. Civ. Eng., 90:69-86.

Marlow, M. S., Lee, H. J., Wright, A. W., 1984. Physical properties of sediment from the Lesser Antilles margin along the Barbados ridge: results from Deep Sea Drilling Project Leg 78A. In Biju-Duval, B., Moore, J. C., et al., Init. Repts. DSDP, 78A: Washington, (U.S. Govt. Printing Office), 549-558.

Mascle, A., Moore, J. C., et al., 1988. Proc. ODP, Init. Repts., 110: College Station, TX (Ocean Drilling Program).

Moore, J. C., and Lundberg, N., 1986. Tectonic overview of DSDP transects of forearcs. In Moore, J. C. (Ed.), Structural Fabrics in DSDP Cores From Forearcs. Mem. Geol. Soc. Am., 166:1-44.

Moore, J. C., Mascle, A., Taylor, E., Andreieff, P., Alvarez, F., Barnes, R., Beck, C., Behrmann, J., Blanc, G., Brown, K., Clark, M., Dolan, J., Fisher, A., Gieskes, J., Hounslow, M., McLellan, P., Moran, K., Ogawa, Y., Sakai, T., Schoonmaker, J., Vrolijk, P., Wilkens, R., and Williams, C., 1988. Tectonics and hydrogeology of the northern Barbados Ridge: results from Ocean Drilling Program Leg 110. Geol. Soc. Am. Bull., 100:1578-1593.

Shephard, L. E., and Bryant, W. R., 1983. Geotechnical properties of lower trench inner-slope sediments. Tectonophysics, 99:279-312.

Shipboard Scientific Party, 1988. Synthesis of shipboard results: Leg 110 transect of the northern Barbados Ridge. In Mascle, A., Moore, J. C., et al., Proc. ODP, Init. Repts., 110: College Station, TX (Ocean Drilling Program), 577-592.

Silva, A., Hetherman, J. R., and Calnan, D. I., 1981. Low gradient permeability testing of fine-grained marine sediments. In Zinnie, T. F., and Riggs, C. O. (Eds.), Permeability and Groundwater Transport: ASTM Spec. Tech. Publ., 746:121-136.

Silva, A., Moran, K., and Akers, S. A., 1983. Stress-strain-time behavior of deep sea clays. Can. Geotech. J., 20:517-531.

Skempton, A. W., 1970. The consolidation of clays by gravitational compaction. J. Geol. Soc. London, 125:373-411. 
Taylor, E., and Bryant, W. R., 1985. Geotechnical properties of sediments from the Middle America Trench and slope. In von Huene, R., Aubouin, J., et al., Init. Repts. DSDP, 84: Washington (U.S. Govt. Printing Office), 745-766.

von Huene, R., and Lee, H. J., 1983. The possible significance of pore fluid pressures in subduction zones. In Watkins, J. S., and Drake, C. L. (Eds.), Studies in Continental Marine Geology, AAPG Mem., 34:781-789.
Westbrook, G. K., and Smith, M. J., 1983. Long décollements and mud volcanoes: evidence from the Barbados Ridge Complex for the role of high pore-fluid pressure in the development of an accretionary complex. Geology, 11:279-283.

Date of initial receipt: 20 December 1988

Date of acceptance: 21 April 1989

Ms 110B-152

\section{Bulk Density $\left(\mathrm{g} / \mathrm{cm}^{3}\right)$}

Atterberg Limits - $W_{p} \circ W_{\mathrm{L}}$
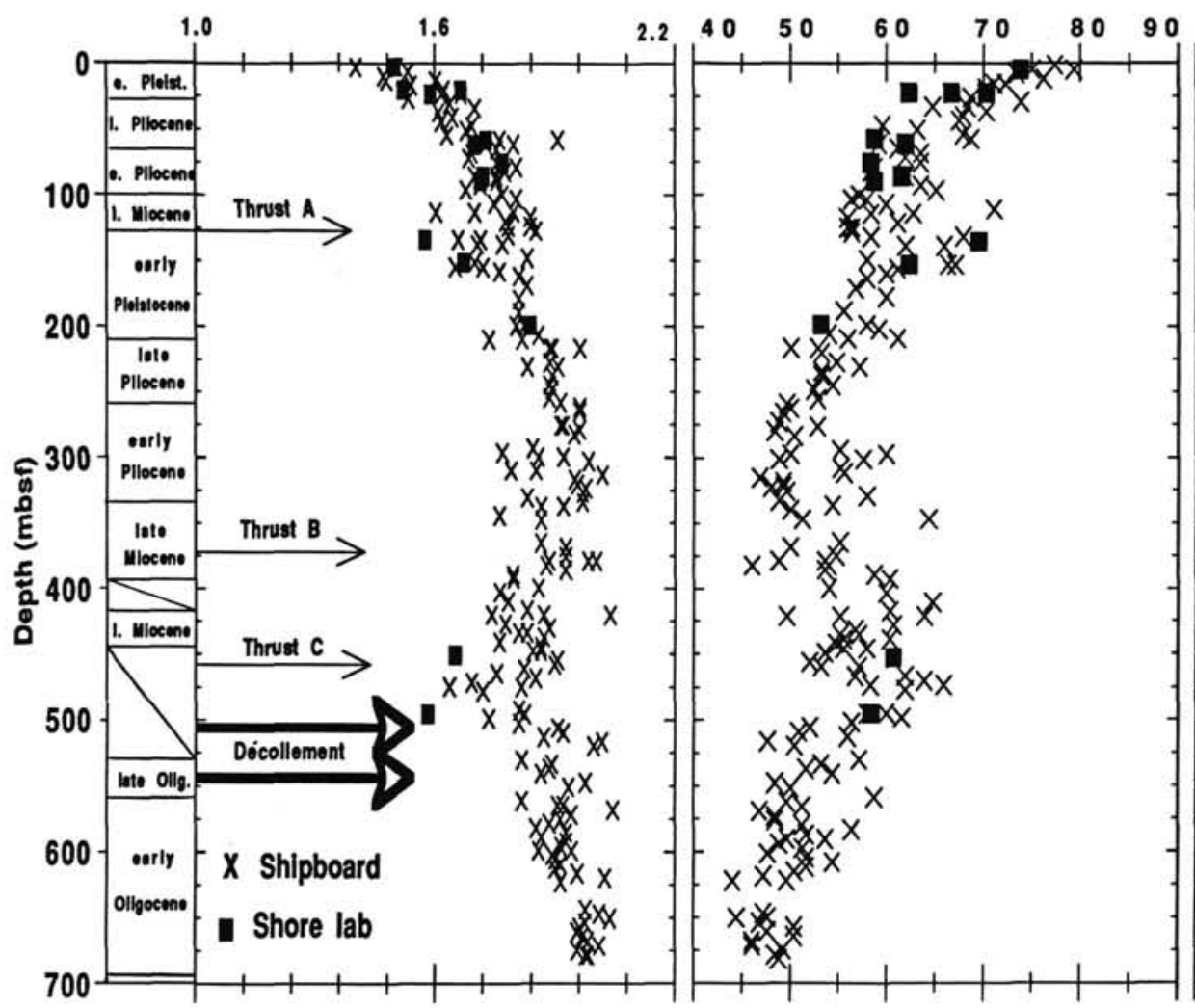

$\begin{array}{lllll}40 & 80 & 120 & 160 & 200\end{array}$

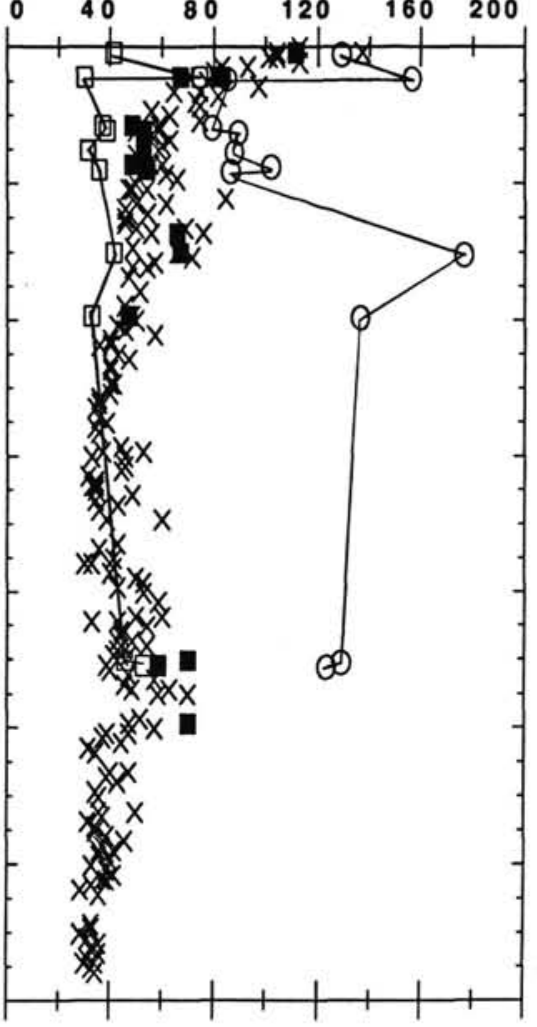

Figure 8. Summary of physical properties vs. depth for Site 671 sediments. 


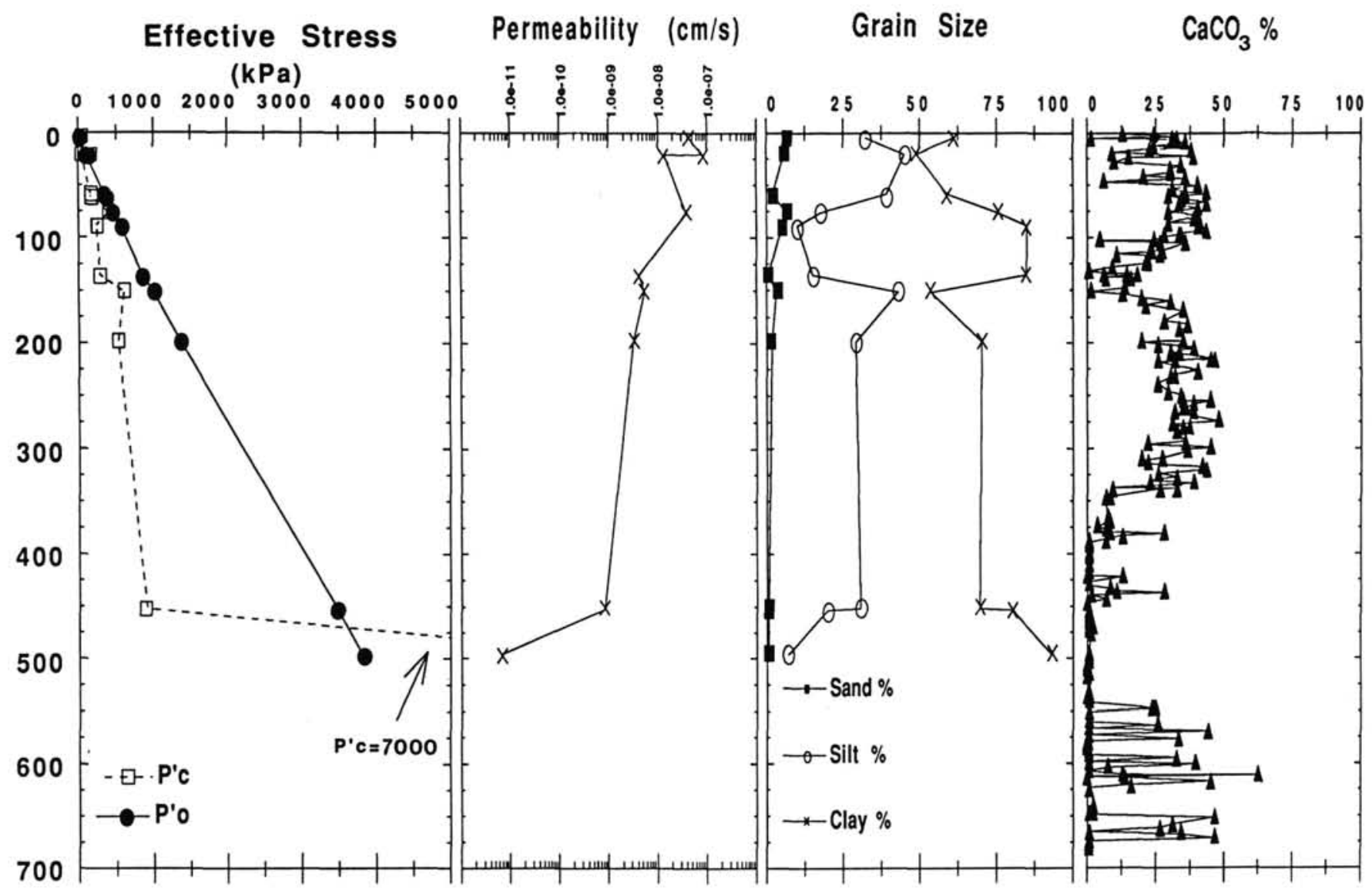

Figure 8 (continued). 

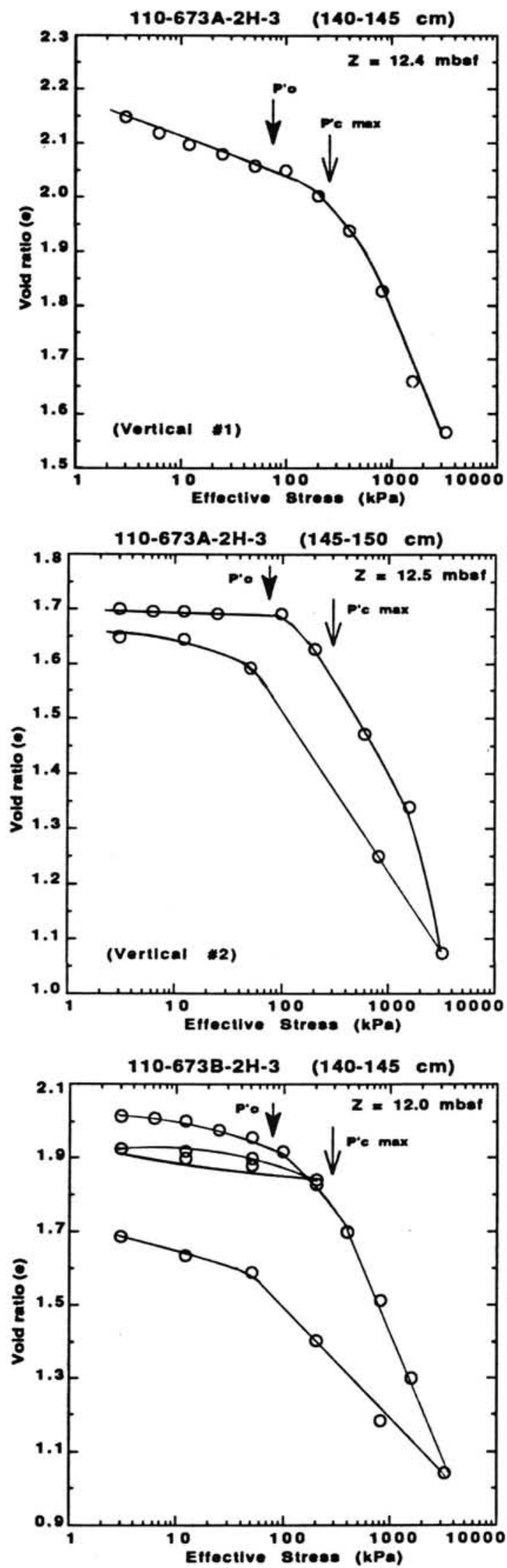

Figure 9. Void ratio vs. effective stress curves for Site 673 consolidation samples. 


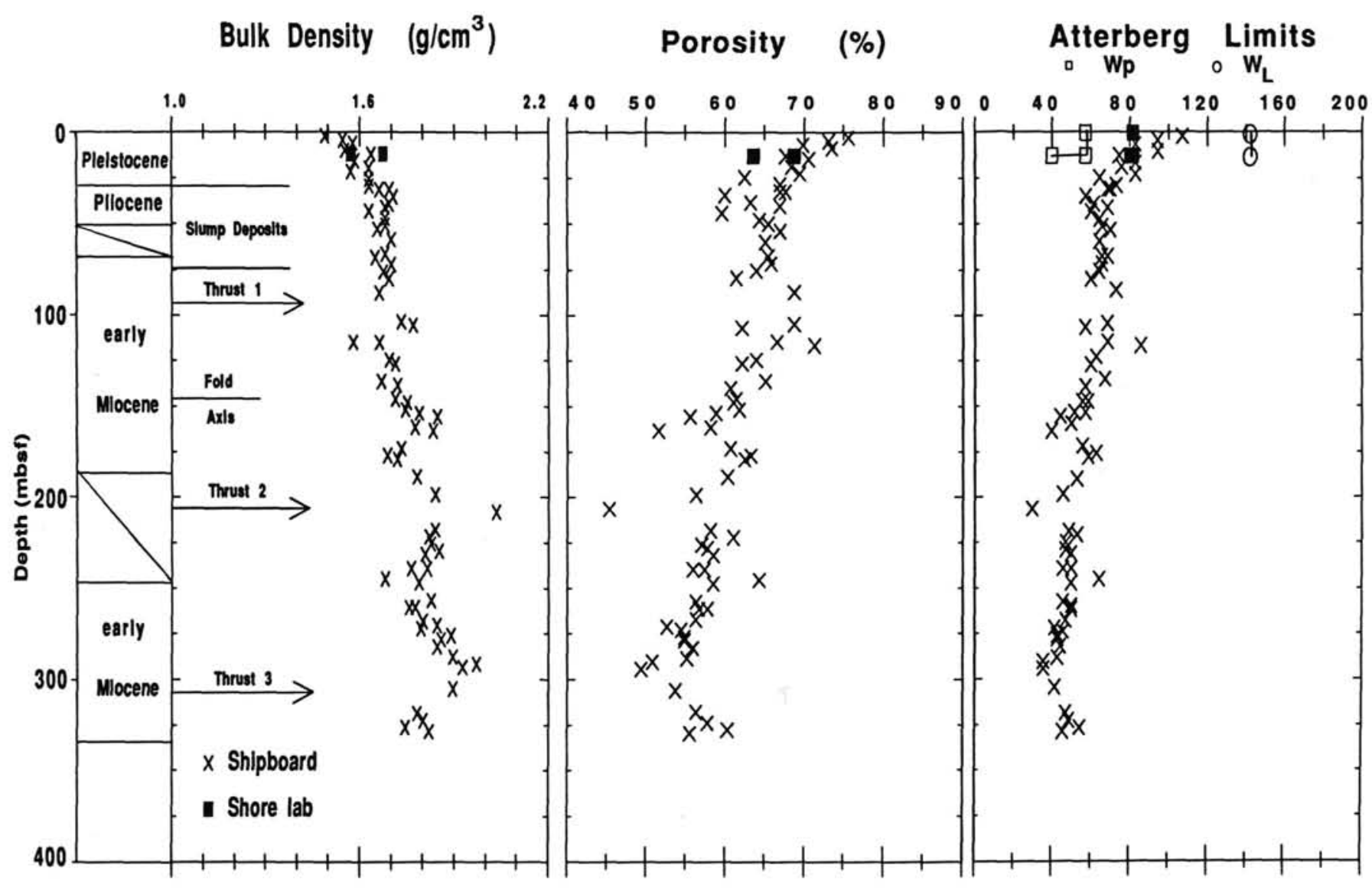

Figure 10. Summary of physical properties vs. depth for Site 673 sediments. 


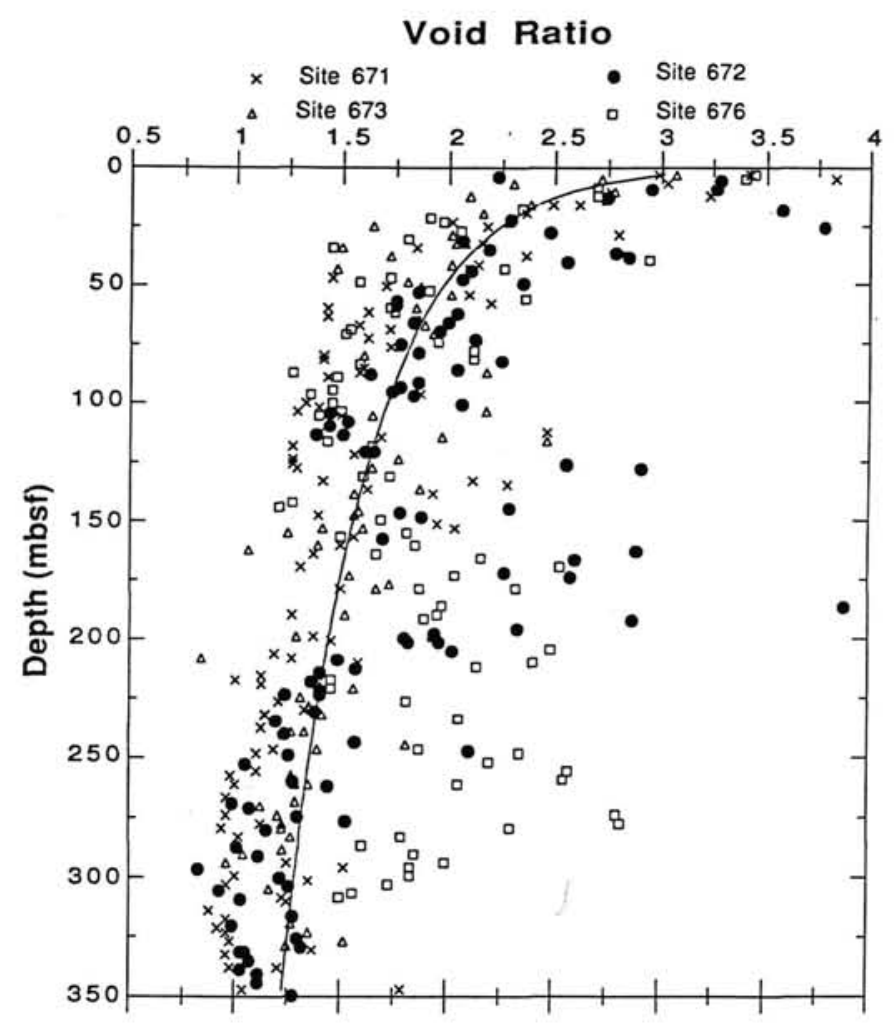

Figure 11. Void ratio at sites across the Barbados convergent margin. The reduction of void ratio in samples from similar sub-bottom depths attests to the effect of lateral compression in consolidation of accreted section. Regression for Site 671: void ratio $=4.4941\left(\right.$ depth $\left.^{-0.22162}\right), \mathrm{r}^{2}$ $=0.66$.

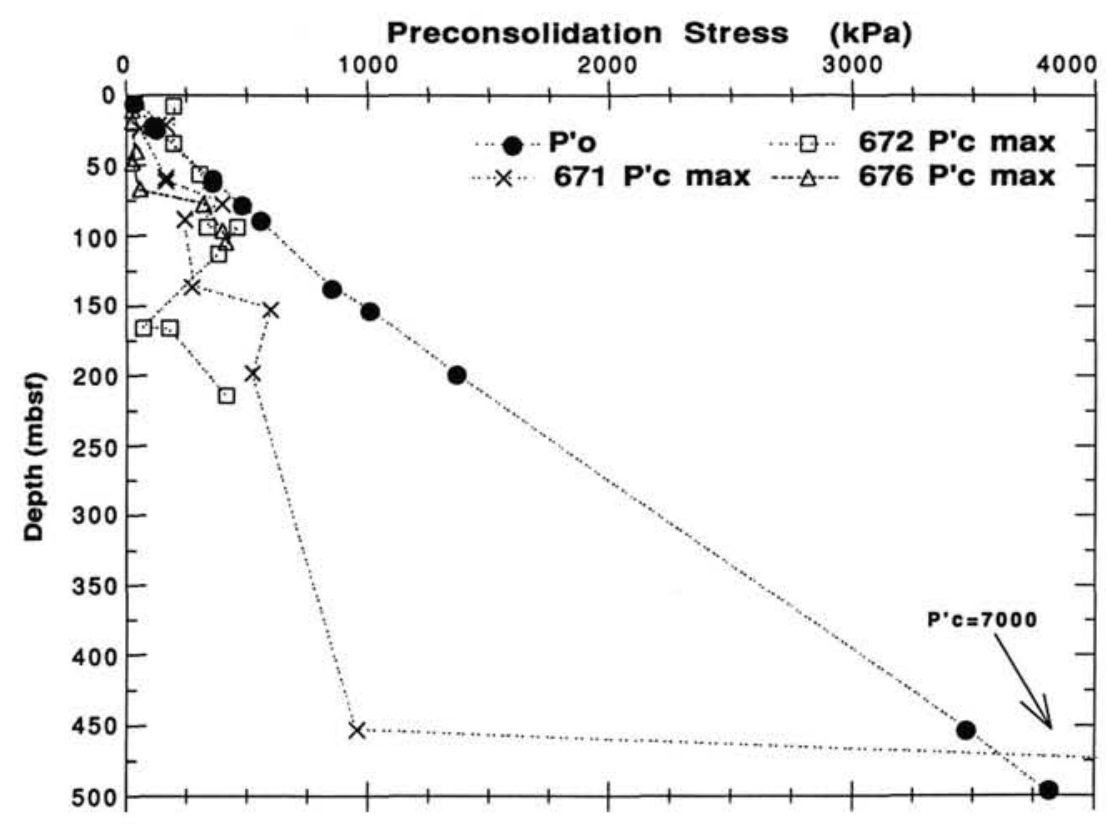

Figure 12. Comparison of preconsolidation stress values for sediments at Sites 672, 676, and 671 . The overburden stress curve $\left(P_{o}^{\prime}\right)$ is drawn from Site 671 data. 


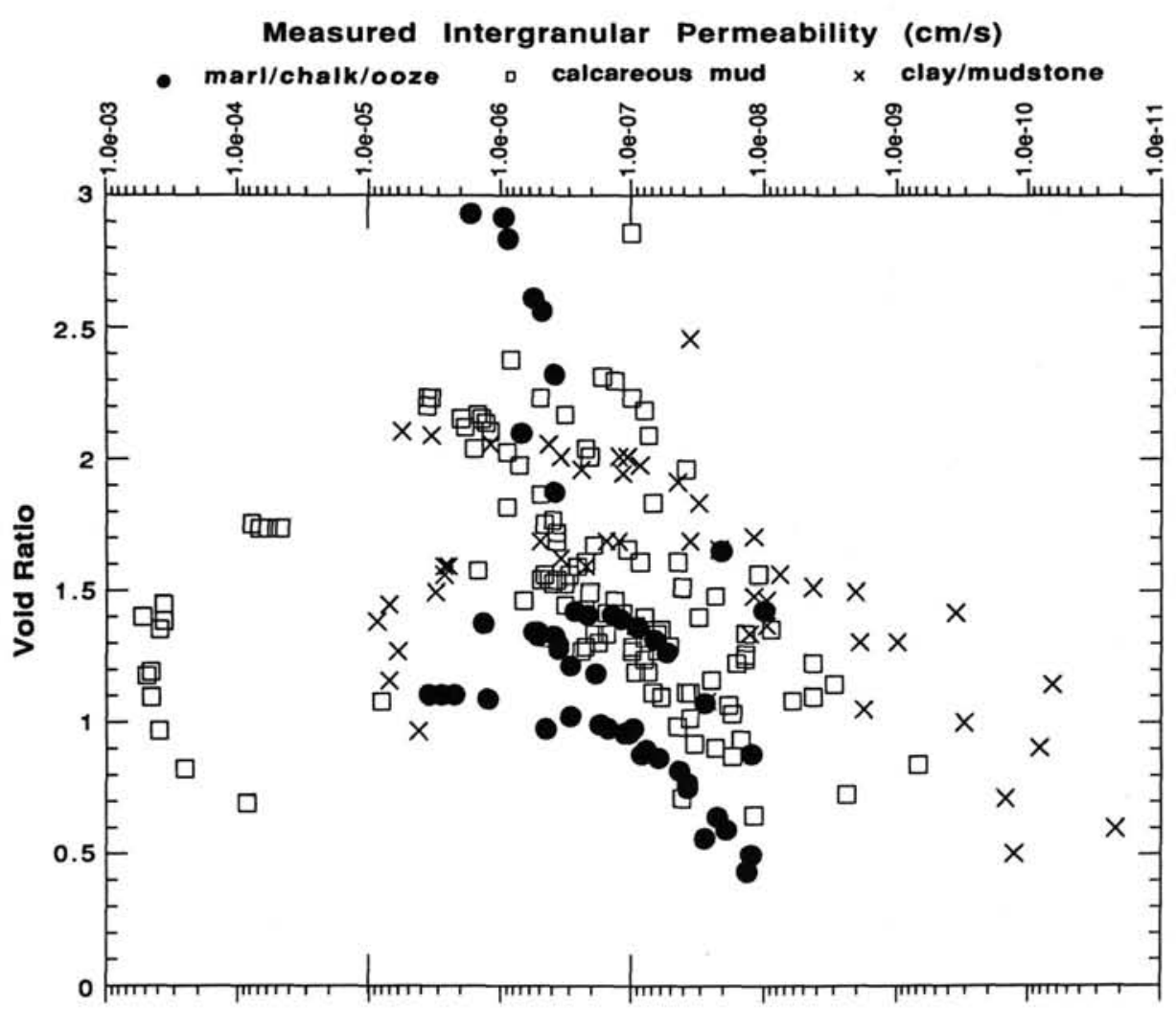

Figure 13. Permeability of Leg 110 lithologies as a function of void ratio (or porosity). 


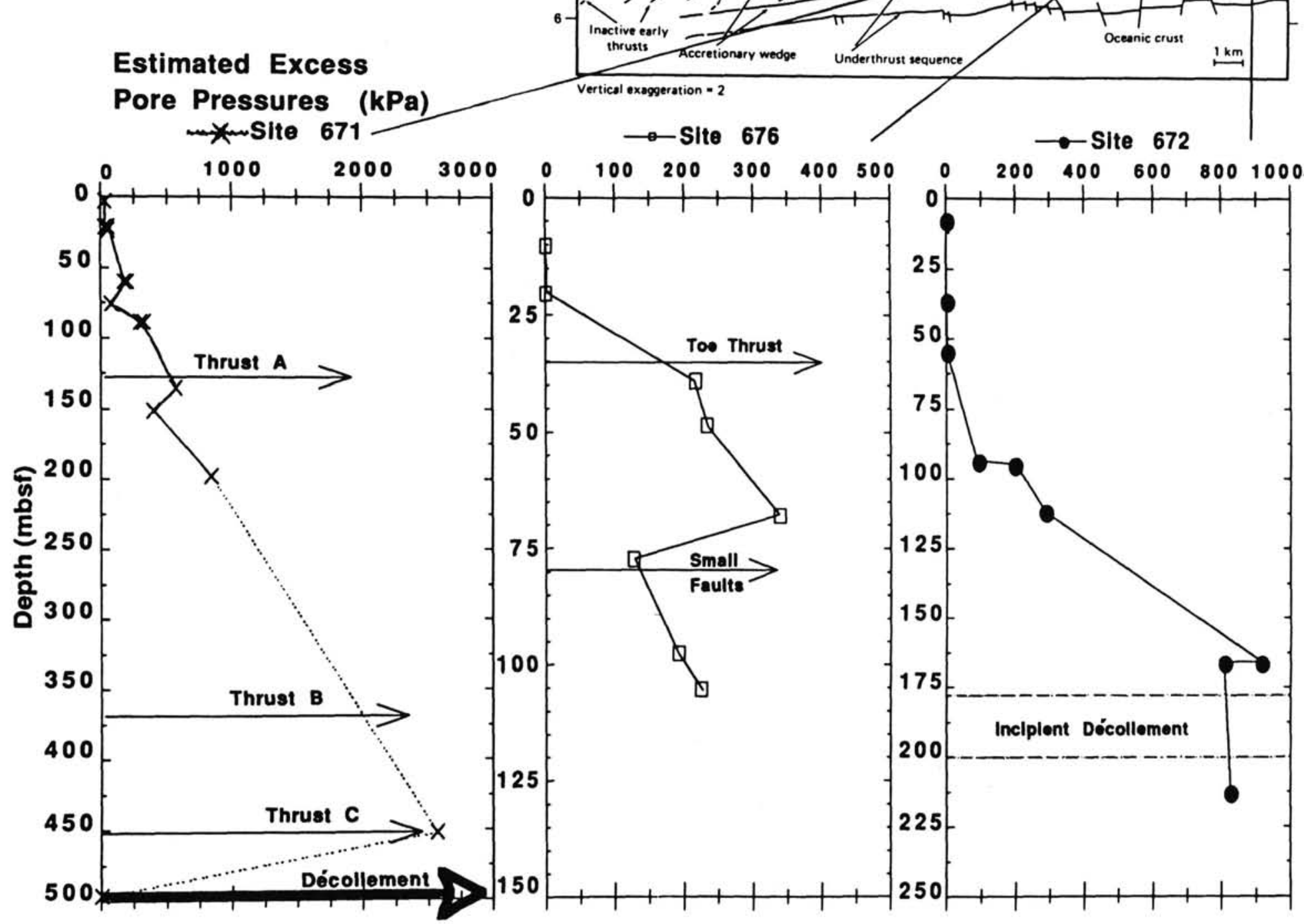

Figure 14. Pore pressures in sediments at the toe of the Barbados forearc are estimated from differences between preconsolidation stress and effective overburden stress. Near-lithostatic pore pressures may exist above the propagating décollement at Site 672. 